\title{
Modeling turbulence-chemistry interaction in lean premixed hydrogen flames with a strained flamelet model
}

\author{
Philipp Trisjono ${ }^{\mathrm{a}, *}$, Konstantin Kleinheinz ${ }^{\mathrm{a}}$, Evatt R. Hawkes ${ }^{\mathrm{b}, \mathrm{c}}$, Heinz \\ Pitsch $^{\mathrm{a}}$ \\ ${ }^{a}$ Institute for Combustion Technology, RWTH Aachen University, 52056 Aachen, \\ Germany \\ ${ }^{b}$ School of Photovoltaic and Renewable Energy Engineering, The University of New \\ South Wales, Sydney, NSW 2052, Australia \\ ${ }^{c}$ School of Mechanical and Manufacturing Engineering, The University of New South \\ Wales, Sydney, NSW 2052, Australia
}

\begin{abstract}
Premixed flames in the thin and broken reaction zones regime exhibit strong non-linear turbulence-chemistry interactions and are hence challenging to model. In the present study, a direct numerical simulation (DNS) database for a hydrogen-air flame is used to understand the effect of turbulencechemistry interactions on the flame speed. To this end, statistics of the local flame front displacement speed are investigated and the roles of flame stretch due to curvature and strain are analyzed. The local flame speed is found to be on average around 30\% lower than the laminar burning velocity, which is shown to be a consequence of strain. Strain effects are then modeled by a recently proposed strained flamelet model, which is validated a priori against the DNS in terms of the flame speed and the reaction source term. The strained flamelet model leads to a moderate improvement of the flame speed prediction and is able to reproduce the decrease of the local flame speed. Moreover, it significantly improves the source term closure, which is demonstrated on the basis of the optimal estimator approach and, overall, describes the investigated DNS flame with very good accuracy.
\end{abstract}

Keywords:

\footnotetext{
${ }^{*}$ Corresponding author

Email address: p.trisjono@itv.rwth-aachen.de (Philipp Trisjono)
}

Preprint submitted to Combust. Flame

July 3, 2016 
DNS, Turbulent combustion, Premixed combustion, Flame speed, Flamelet modeling

\section{Introduction}

Turbulent premixed combustion of high hydrogen content fuels is a phenomenon of considerable practical interest in a variety of modern energy systems, particularly in stationary gas turbines. In this context, hydrocarbon fuels mixed with significant amounts of hydrogen are becoming increasingly important. For instance, hydrogen production from renewable resources could play an increased future role as energy storage that might be added to the natural gas supply. Another example is the gasification of solid fossil fuels. This supports cleaner combustion and carbon capture and storage technologies. To improve the efficiency of industrial devices, reduce their pollutant emissions, and ensure their stable operation, a comprehensive understanding of the underlying phenomena and accurate yet affordable models for premixed hydrogen combustion are essential.

A series of modeling frameworks for turbulent premixed combustion has been proposed, including the probability density function transport equation model [1], the thickened flame model [2], the linear eddy model [3], the conditional moment closure concept [4], the flamelet approach [5], and the flame surface density model [6, 7]. Additionally, manifold methods, such as flamelet generated manifolds (FGM) [8] and the flame prolongation of intrinsic lower dimensional manifolds (ILDM) referred to as the FPI framework [9] are often used in conjunction with some combustion models. The present article investigates strained and unstrained flamelet models, which map solutions of one-dimensional flamelet equations into a three-dimensional field and combine asymptotic ideas of the flamelet concept with the manifold approach.

Premixed flames in the thin reaction zones regime are particularly challenging to model, since mixing is strong enough for turbulent eddies to enter the preheat zone and to perturb the flame structure [10]. One primary attribute of flames in the thin reaction zones regime is the presence of strong stretch effects in form of curvature and strain. A number of combustion models $[11,12]$ that have mainly been developed within the FGM or FPI framework, seek to describe how premixed flame structures are perturbed by flame stretch. Van Oijen et al. [12] performed DNS of turbulent premixed freely 
expanding flames and analyzed how various sets of two-dimensional flamelet generated manifolds could predict the results. The FGMs were created in three different ways. First, unstretched premixed flamelets at varying initial compositions were used. The two more variants of FGMs were computed from flamelets submitted to a constant stretch rate and constant curvature, respectively. Interestingly, they found that a two-variable parameterization of premixed flamelets is able to account for stretch and curvature effects independently of the way the FGMs were constructed. The difference to the strained flamelet model proposed by Knudsen et al. [13], which is further analyzed in the present work, is that different types of flamelet equations are considered. While the model of Knudsen et al. relies on strained premixed flamelets, Van Oijen et al. derive flamelet equations that explicitly contain a stretch and a curvature term.

Compared to unstretched laminar flamelets, strained flamelet solutions have been suggested as an appealing means to model flames in the thin reaction zones regime that are submitted to strong flame stretch. A long standing question has been whether back-to-back or fresh-to-burnt flamelets are more suited to describe these flames. Hawkes and Chen [14] have compared strained flamelets from both configurations to lean methane-air flames of a DNS and found that especially for high strain rates fresh-to-burnt flamelets provide a much improved prediction of the displacement speed in comparison to back-to-back flamelets. In Kolla and Swaminathan [15], strained flamelets were used for a RANS (Reynolds averaged Navier-Stokes) simulation of laboratory flames, where fresh-to-burnt flamelet solutions were incorporated into the simulation by parameterizing these flamelets in terms of the scalar dissipation rate of the progress variable. In their choice of the flamelet configuration they followed Hawkes and Chen [14] and argued that fresh-to-burnt flamelets are more representative of practical combustion situations. Recently, Knudsen et al. [13] have proposed a strained flamelet model in the context of large-eddy simulation (LES) that leads to very good results in predictions of a DNS data set for an methane-air Bunsen flame. The key idea behind this model is to use a species as the strain marker and to parameterize strained flamelets by this species. Knudsen and coworkers found that this parameterization removes the arbitrariness of selecting a particular flamelet configuration, since back-to-back and fresh-to-burnt flamelet profiles collapse when parameterized by an appropriate species.

The objective of the present paper is to analyze and model turbulencechemistry interactions in a temporally developing premixed jet flame DNS [16] 
in the thin reaction zones regime. This flame has been observed to propagate notably different than a laminar unstretched flame and to be subject to strong finite-rate chemistry effects that are not understood or modeled yet. In the first part of this study, the reported finite-rate chemistry effects are investigated by means of a flame front displacement analysis, while in the second part, the strained flamelet model of Knudsen et al. [13] is utilized and validated against the DNS database. The model validation is accompanied by a discussion regarding capabilities and limitations of the aforementioned model.

The paper is organized as follows. In Sect. 2, the DNS database is described. Next, in Sect. 3 a comprehensive flame front displacement speed analysis is presented that considers statistics of the flame front displacement speed and analyzes the influence of flame stretch. Sect. 4 picks up implications of the flame front displacement analysis and reformulates a strained flamelet model in order to account for flame stretch. The strained flamelet model is assessed a priori in Sect. 5. Important conclusions are summarized in Sect. 6.

\section{Description of DNS}

Hawkes et al. [16] have carried out a peta-scale DNS of lean hydrogen-air flames, where a parametric variation of the Damköhler number at a fixed jet Reynolds number of $\operatorname{Re}_{j}=10000$ was realized. The data of these flames were used to develop a fractal model for the turbulent burning velocity for LES and to explore fractal properties of low Damköhler number flames [17]. In the present paper, the flame case "Da-" is analyzed as it features the highest Karlovitz number Ka among the available DNS cases and is hence characterized by the strongest turbulence-chemistry interactions of these DNS flames. It is therefore the most challenging and relevant flame case for the present study.

\subsection{DNS configuration}

In the DNS, two initially laminar flames propagate into a temporally developing jet of unburnt premixed reactants with an unburnt temperature of $T_{u}=700 \mathrm{~K}$, an equivalence ratio of $\phi=0.7$, and a pressure of one atmosphere as illustrated in Fig. 1. The trajectory of the flame in a regime diagram for

premixed combustion is shown in Fig. 1, where peak Ka and Re numbers of 92 and 810 are achieved, respectively. Note that the regime diagram has been 
adjusted to account for the fact that for the present conditions, $s_{L} l_{F} / \nu_{u} \approx 50$, which is substantially different from unity as typically assumed [10]. $s_{L}$ and $l_{F}$ denote the laminar burning velocity and flame thickness associated with the DNS operating conditions based on the temperature gradient, and $\nu_{u}$ is the viscosity of the fresh gases. An overview of the operating conditions of the DNS is provided in Tab. 1 , where $U_{j}$ and $H_{j}$ denote the jet velocity and width, respectively, that can be used to define a normalized jet time $t_{j}=H_{j} / U_{j}$.
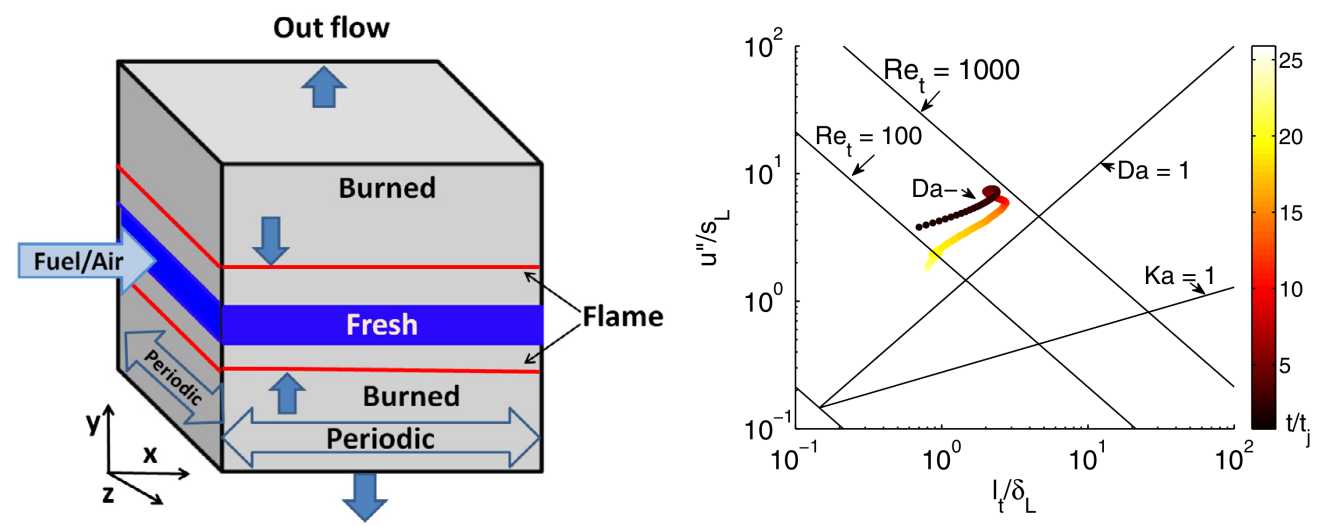

Figure 1: Left: Configuration. Right: Trajectory of flame case Da- in a regime diagram colored by normalized time.

\begin{tabular}{ccccc}
$\operatorname{Re}_{j}$ & $U_{j}[\mathrm{~m} / \mathrm{s}]$ & $H_{j}[\mathrm{~mm}]$ & $\phi$ & $T_{u}[\mathrm{~K}]$ \\
\hline 10000 & 312.6 & 2.7 & 0.7 & 700
\end{tabular}

Table 1: DNS conditions of flame case Da-.

The simulation has been conducted with the massively parallel Sandia DNS code S3D [18] on the Jaguar Cray XT5 Supercomputer at Oak Ridge National laboratories using 120000 cores. Non-reflecting boundary conditions are applied in the lateral direction to allow for the burnt products to exit the domain, while the streamwise and spanwise directions are periodic. The computational domain is discretized with $2400 \times 1600 \times 1800 \approx 7$ billion grid points, which span a physical domain of $16 H_{j} \times 20 H_{j} \times 12 H_{j}$. Chemical reactions are described by a reduced mechanism for the oxidation of hydrogen that consists of 9 species and 21 reactions [19]. It is emphasized that both 
flame and turbulence are very well resolved. The laminar flame thickness $l_{F}$ is resolved by 28 points, while the Kolmogorov scale $\eta$ is resolved by a minimum of 0.6 grid points in the region of interest. These numbers satisfy canonical resolution criteria and highlight the fidelity of the DNS database. The analyses presented in Sects. 3, 5.1, and 5.2 are carried out at the normalized time $t / t_{j}=17.1$, which is the time of maximum heat release and thus, the instant of strongest turbulence-chemistry interactions. For further details regarding the DNS the reader is referred to [16].

\subsection{Global burning rate statistics}

A main requirement of combustion models is the accurate prediction of the total heat release rate. Many modeling frameworks for premixed flames, for example the coupled level set progress variable flamelet model [20], decompose this task into two components. These parts are the prediction of the flame surface area on the one hand, and modeling of the local flame speed on the other hand, which is one of the modeling targets of the present study.

In order to estimate the burning rate per unit area with respect to a laminar unstretched flame, a correction factor $I_{0, \mathrm{H}_{2} \mathrm{O}}$ can be introduced [21], which is defined as the ratio of the production speed $s_{p, \mathrm{H}_{2} \mathrm{O}}$ of $\mathrm{H}_{2} \mathrm{O}$ and the flame surface area ratio $\sigma_{\mathrm{H}_{2} \mathrm{O}}$ multiplied by the laminar burning velocity

$$
I_{0, \mathrm{H}_{2} \mathrm{O}}=s_{p, \mathrm{H}_{2} \mathrm{O}} /\left(s_{L} \sigma_{\mathrm{H}_{2} \mathrm{O}}\right) \text {. }
$$

If the entire change of heat release compared to a laminar unstretched flame arises from a change of the flame surface area, $I_{0, \mathrm{H}_{2} \mathrm{O}}$ is unity. Otherwise, $I_{0, \mathrm{H}_{2} \mathrm{O}}$ indicates a departure of the net flame speed from the laminar burning velocity. In Eq. 1, $s_{p, \mathrm{H}_{2} \mathrm{O}}$ is the integrated production speed of $\mathrm{H}_{2} \mathrm{O}$, which for the present case can be defined as

$$
s_{p, \mathrm{H}_{2} \mathrm{O}}=\frac{1}{\rho_{u}\left(Y_{\mathrm{H}_{2} \mathrm{O}, b}-Y_{\mathrm{H}_{2} \mathrm{O}, u}\right)} \int_{0}^{L_{y}}\left\langle\dot{m}_{\mathrm{H}_{2} \mathrm{O}}\right\rangle \mathrm{d} y,
$$

where $\rho_{u}$ denotes the unburnt density, $L_{y}$ the extent of the domain in the transverse direction, and $\dot{m}_{\mathrm{H}_{2} \mathrm{O}}$ the chemical source term of $\mathrm{H}_{2} \mathrm{O} . Y_{\mathrm{H}_{2} \mathrm{O}}$ is the mass fraction of $\mathrm{H}_{2} \mathrm{O}$ and the indices $\mathrm{b}$ and $\mathrm{u}$ refer to burnt and unburnt conditions, respectively. The $\langle\cdot\rangle$ operator denotes spatial averaging in streamwise and spanwise directions. In addition, the symmetry in the ydirection is taken advantage of by averaging all statistics over the upper and 
lower sections of the domain. The flame surface area ratio $\sigma_{\mathrm{H}_{2} \mathrm{O}}$ appearing in Eq. 1 is given by an integral of a generalized flame surface density [6]

$$
\sigma_{\mathrm{H}_{2} \mathrm{O}}=\int_{0}^{L_{y}}\langle|\nabla C|\rangle \mathrm{d} y,
$$

where the normalized progress variable $C$ is defined as

$$
C=\frac{Y_{\mathrm{H}_{2} \mathrm{O}}-Y_{\mathrm{H}_{2} \mathrm{O}, u}}{Y_{\mathrm{H}_{2} \mathrm{O}, b}-Y_{\mathrm{H}_{2} \mathrm{O}, u}}
$$

Note that in contrast to the original definition of Peters [5], the above definition of the flame surface area ratio is not conditioned on a particular isosurface, which is attractive due to its straightforward calculation. To understand the impact of this simplification, the flame surface area of the inner layer isosurface $C=0.5$ and the value according to Eq. 3 have been compared at the time of maximum heat release, which revealed a difference of less than $5 \%$. Therefore, $I_{0, \mathrm{H}_{2} \mathrm{O}}$ based on the flame surface area ratio $\sigma_{\mathrm{H}_{2} \mathrm{O}}$ using Eq. 3 is regarded an appropriate estimate for the integrated flame speed and the impact of finite-rate chemistry effects.

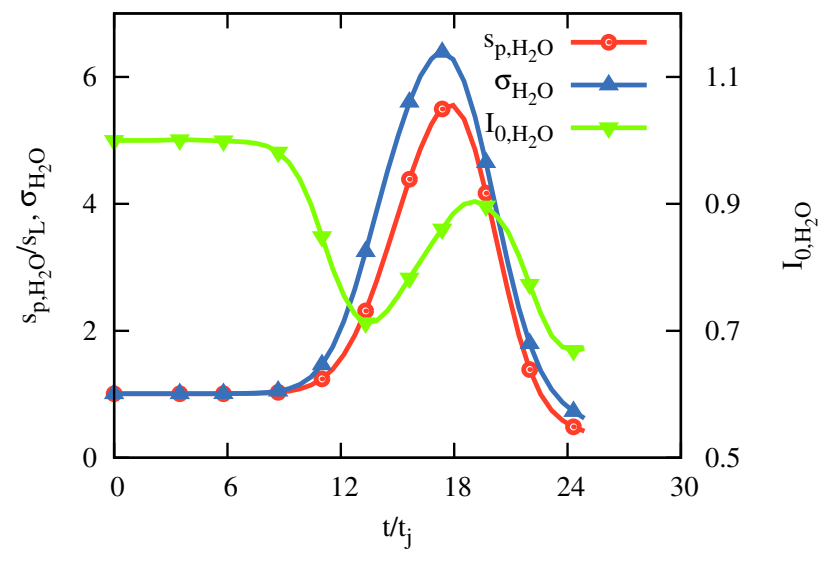

Figure 2: Integrated production rate of $\mathrm{H}_{2} \mathrm{O}$ (red), flame surface area ratio (blue), and correction factor $I_{0, \mathrm{H}_{2} \mathrm{O}}$ (green) that converts laminar burning rate per unit area into turbulent burning rate per unit area.

The quantities $s_{p, \mathrm{H}_{2} \mathrm{O}}, \sigma_{\mathrm{H}_{2} \mathrm{O}}$, and $I_{0, \mathrm{H}_{2} \mathrm{O}}$ are shown in Fig. 2 as a function of normalized time. While the production of $\mathrm{H}_{2} \mathrm{O}$ follows the principal trend of the flame surface area, the increase in $\mathrm{H}_{2} \mathrm{O}$ production is lower than the 
flame surface area ratio, which results in $I_{0, \mathrm{H}_{2} \mathrm{O}}$ becoming less than unity. This behavior indicates the need for modeling of the flame speed. Besides finite-rate chemistry effects it can also originate from other causes such as effects of unsteadiness.

Finally, it is remarked that the $I_{0, \mathrm{H}_{2} \mathrm{O}}$ factor based on $\mathrm{H}_{2} \mathrm{O}$ shown here deviates significantly from $I_{0, \mathrm{H}_{2}}$ based on $\mathrm{H}_{2}$ that has been discussed by Hawkes et al. [16]. It differs not only in its absolute value, but also shows a different trend, as $I_{0, \mathrm{H}_{2} \mathrm{O}}$ is less than unity once the flame hits the turbulence, while $I_{0, \mathrm{H}_{2}}$ is greater than unity. Since the production speed of $\mathrm{H}_{2} \mathrm{O}$ and consumption speed of $\mathrm{H}_{2}$ are almost equal, this difference is attributed to different flame surface area ratios of $\mathrm{H}_{2} \mathrm{O}$ and $\mathrm{H}_{2}$, which in turn can be attributed to the larger diffusivity of $\mathrm{H}_{2}$. The non-linearity of $I_{0, \mathrm{H}_{2} \mathrm{O}}$ is not addressed in this article and remains an open question for future work. However, both $I_{0, \mathrm{H}_{2} \mathrm{O}}$ and $I_{0, \mathrm{H}_{2}}$ have in common that they are non-linear. The non-linearity of $I_{0, \mathrm{H}_{2}}$ has already been discussed in Ref. [16], which might serve as a basis for future investigation of the non-linearity of $I_{0, \mathrm{H}_{2} \mathrm{O}}$.

\section{Flame front displacement analysis}

Premixed flames in the thin reaction zones regime contain eddies that are smaller than the flame thickness. These are able to enter the preheat zone and perturb the flame structure. Thus, molecular transport between the reaction zone and the upstream preheat zone is an unsteady process, where the notion of a laminar burning velocity $s_{L}$ of a laminar flame has less relevance than in the corrugated flamelet regime. Instead however, a flame front displacement speed, describing the motion of an isosurface [22, 23], can be introduced. It represents the local propagation of a turbulent premixed flame in the thin reaction zones regime.

A number of previous studies has investigated various aspects of the flame front displacement speed including the analysis of global statistics of the flame front displacement speed $[24,25]$ as well as the determination of the effects of flame stretch [26, 27, 28, 29, 30], different fuels [31], the Karlovitz number [32], and flame instabilities [33] on the flame front displacement speed. Several of the features found in the following analysis have already been observed in previous studies, e. g. Sankaran and coworkers report a very similar response of the flame front displacement speed to the strain rate [34]. In this section, the flame front displacement speed is analyzed with respect 
to flame stretch resulting from curvature and strain, so that the combustion model in Sect. 4 can be formulated accordingly.

\subsection{Mathematical formulation}

From a balance equation of an appropriate chemical component or the temperature, the motion of an isosurface can be tracked and a corresponding displacement speed $s_{d}$ can be identified. In this work, the displacement of a progress variable isosurface is considered. Key results of the subsequent analysis of the flame front displacement speed are also studied in terms of a generalized flame speed in Sect. 3.3.4.

The flame displacement speed $s_{d}$ constitutes of terms due to tangential diffusion, normal diffusion, and reaction and adds up as

$$
s_{d}=\mathcal{D} \kappa+V_{n}+V_{r} .
$$

$\mathcal{D} \kappa$ is the tangential diffusion term, $\mathcal{D}$ the diffusivity of the progress variable and $\kappa$ denotes the curvature that is the divergence of the normal vector $\mathbf{n}$ pointing towards the burnt gases

$$
\mathbf{n}=\frac{\nabla C}{|\nabla C|}
$$

Given this definition, positive curvature is associated with flame regions that are convex towards the burnt gases, while negative curvature corresponds to flame pockets that are convex towards the unburnt gases. $V_{n}$ and $V_{r}$ are the contributions due to normal diffusion across the isosurface and reaction, respectively, which are given by

$$
V_{n}=\frac{\mathbf{n} \cdot \nabla(\rho \mathcal{D} \mathbf{n} \nabla C)}{\rho|\nabla C|}
$$

and

$$
V_{r}=\frac{\dot{m}_{C}}{\rho|\nabla C|},
$$

where $\rho$ denotes the density and $\dot{m}_{C}$ the source term of the progress variable. Note that in case of a laminar unstretched flame, the displacement speed $s_{d}$ is equal to the laminar burning velocity $s_{L}$ evaluated at the same progress variable isosurface, $s_{L, 0}$ (defined below).

Figs. 3(a) and 3(b) show a three-dimensional snapshot of the isosurface $C=0.5$ and a two-dimensional plane view of the isosurfaces $C=0.1, C=$ 


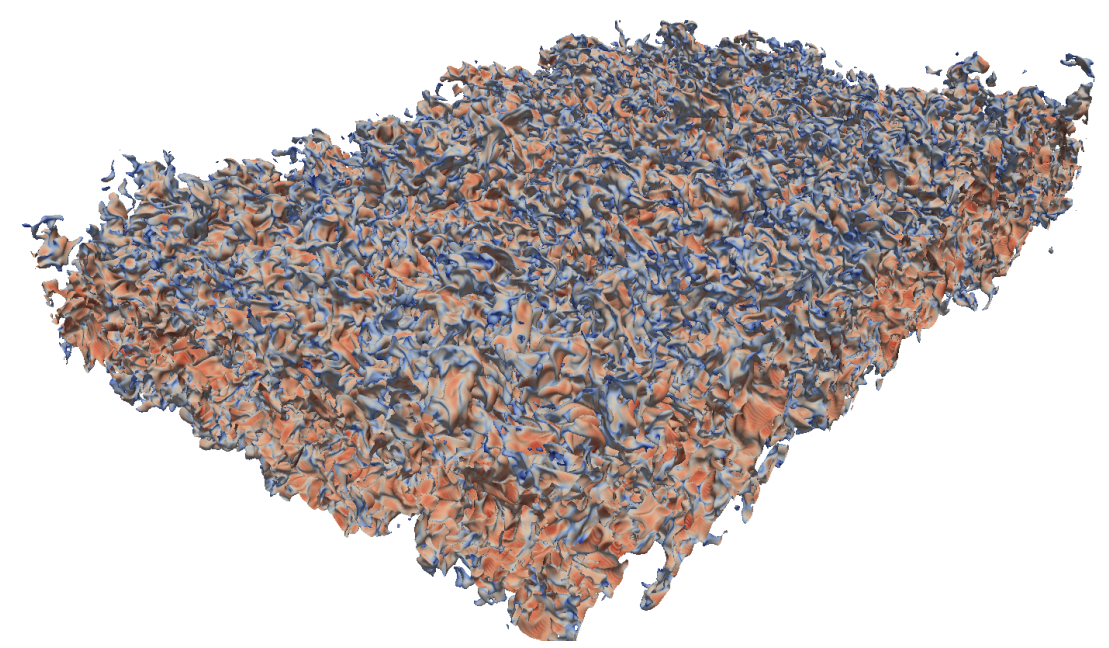

(a) 3D snapshot of the isosurface $C=0.5$ colored with the scalar dissipation rate of the progress variable. Isosurface that was used to collect statistics of the displacement speed $s_{d}$. A high resolution image is provided in the supplementary material.

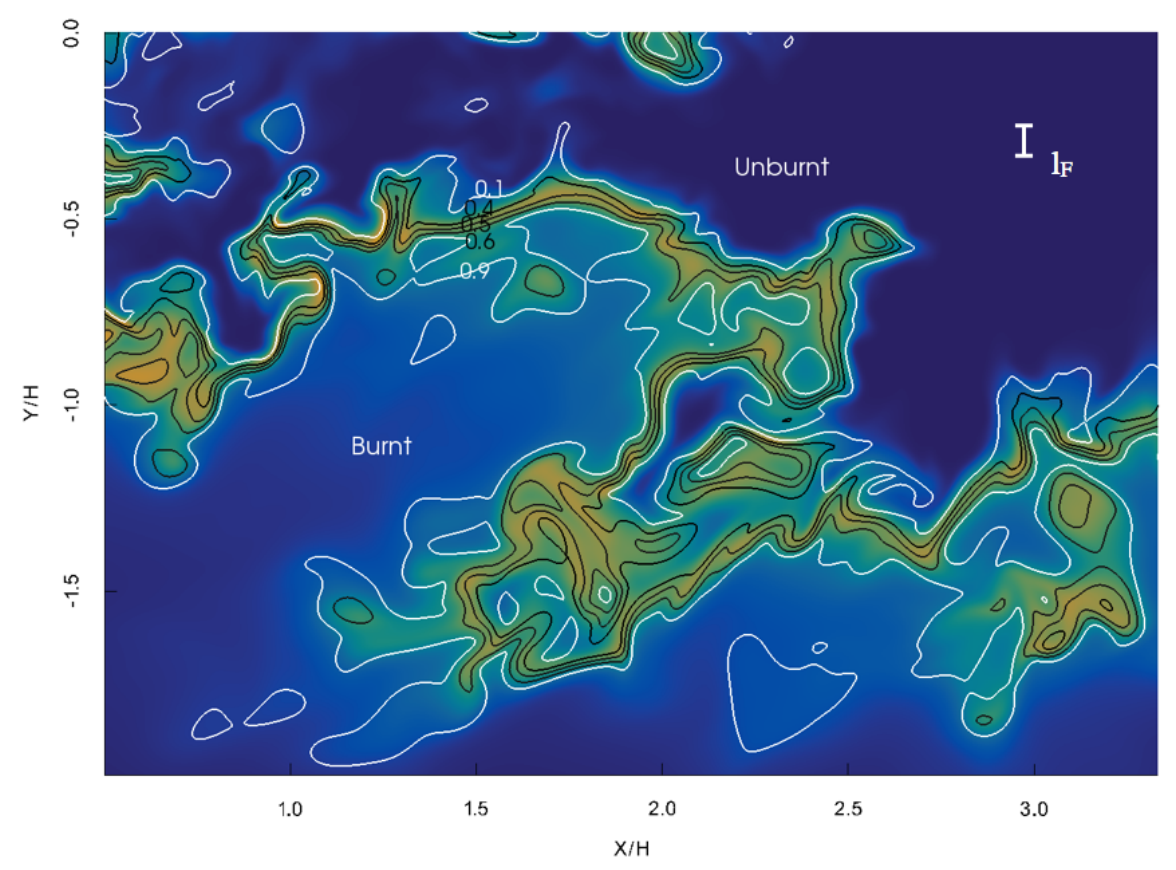

(b) 2D snapshot of the heat release overlaid with the isolines $C=0.1, C=0.4$, $C=0.5, C=0.6$, and $C=0.9$. Contour plot of the heat release normalized with the maximum heat release from an unstretched premixed flame with three different progress variable isolines.

Figure 3: Visualization of the flame at the time of maximum total heat release $t / t_{j}=17.1$. 
$0.4, C=0.5, C=0.6$, and $C=0.9$ that track the heat release. Visual inspections of instantaneous fields of heat release and potential candidates to track the flame revealed that $\mathrm{H}_{2} \mathrm{O}$ follows the heat release more closely than other species such as for example $\mathrm{H}_{2}$. Fig. $3(\mathrm{~b})$ reveals that the heat release occurs in a relatively thin reaction zone without any flame extinction as theoretically expected in the thin reaction zones regime that the investigated flame sits in. Further, a broadening is observed towards both the unburnt region corresponding to $C=0.1$ and the burnt region corresponding to $C=$ 0.9. In the remainder of this study, the isosurface $C=0.5$ is selected for the flame front displacement analysis and the sensitivity of $s_{d}$ to this choice and to the progress variable definition is discussed in supplementary material. The flame displacement speed and its contributions are normalized by the density weighted laminar burning velocity

$$
s_{L, 0}=s_{L, u} \frac{\rho_{u}}{\rho_{0}}
$$

where $\rho_{0}$ is the density at $C=0.5$ of the laminar unstretched flame. It is noted that the relationship in Eq. 9 is satisfied for expansion across a onedimensional planar flame. In the DNS, the flame is not one-dimensional, and flow divergence within the flame would be affected by curvature and indeed by the large-scale pressure field. Therefore, this density correction is only approximate.

\subsection{Statistical evaluation}

\subsubsection{Displacement speed PDFs}

Probability density functions (PDFs) of the individual contributions of $s_{d}$ are shown on the left of Fig. 4. The curvature contribution exhibits a narrow shape and is almost symmetric around zero. While the reaction contribution $V_{r}$ is strictly positive, the normal diffusion term $V_{n}$ is mostly negative and has a negative mean value.

Fig. 4 also shows the PDFs of $s_{d}$ and the sum of normal diffusion and reaction contribution. These PDFs lead to two important conclusions. The first one is that the PDF of $s_{d}$ implies a mean value of $\left\langle s_{d} / s_{L, 0}\right\rangle=0.66$, which means that the flame locally burns slower than its laminar unstretched counterpart. This result is in agreement with the theoretical picture outlined by Peters [10] in a sense that the averaged local flame speed is of the order of the laminar burning velocity. Peters argued that as the curvature has a mean of approximately zero, the tangential diffusion component's contribution is 

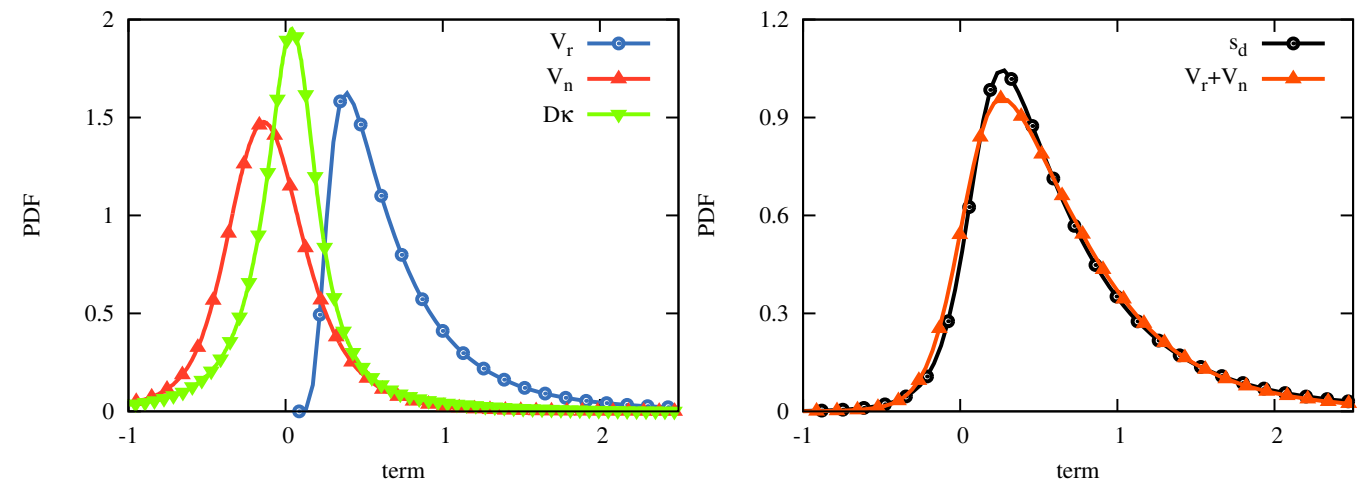

Figure 4: Left: PDFs of $\mathcal{D} \kappa$ (green), $V_{n}$ (red), and $V_{r}$ (blue). Right: PDFs of $V_{n}+V_{r}$ (orange) and $s_{d}$ (black). All terms are normalized with the laminar burning velocity $s_{L, 0}$.

also approximately zero. He argued that the PDF of $s_{d}$ and the averaged $\left\langle s_{d} / s_{L, 0}\right\rangle$ are therefore principally a result of the normal diffusion and reaction components. This is supported by a comparison of the PDFs of $s_{d}$ and the sum of $V_{r}$ and $V_{n}$ in the right panel of Fig. 4, which shows that these are almost equal. Peters further argued that the normal diffusion and reaction components are also only weakly affected by turbulence on average, since the frequencies of turbulent straining are too high for the flame to respond. Based on these arguments he concluded that the local flame speed on average is of the order of the laminar burning velocity, while quantitatively differences between the averaged flame speed and the laminar burning velocity may exist due to other effects of higher order.

The finding of a decreased burning velocity is further consistent with the analysis presented in Sect. 2.2. Although $I_{0, \mathrm{H}_{2} \mathrm{O}}$ and $\left\langle s_{d} / s_{L, 0}\right\rangle$ are different in a sense that $I_{0, \mathrm{H}_{2} \mathrm{O}}$ is an unconditioned quantity, while $\left\langle s_{d} / s_{L, 0}\right\rangle$ is associated with a particular isosurface, they indicate the same trend of a decreased burning rate. Furthermore, it is remarked that $s_{d}$ occasionally becomes negative in regions of the flame that feature negative $V_{n}$ and low values of $V_{r}$. These rare events are suggested to originate from unsteady mixing between the preheat and the reaction zone and are triggered by strong finite-rate chemistry effects.

\subsection{Influence of flame stretch}

Flame stretch is one important consequence of turbulence-chemistry interactions and has previously been found to be responsible for significant 
differences between $s_{d}$ and the laminar burning velocity [25]. Therefore, the response of $s_{d}$ to curvature and strain and their interdependencies are analyzed in the following. The non-dimensional stretch rate is given by

$$
\mathcal{K}=a \tau_{F}+\kappa l_{F}
$$

where $\tau_{F}=l_{F} / s_{L, u}$ is the flame time. The strain rate $a$ is defined as

$$
a=-\mathbf{n} \cdot \nabla \mathbf{u} \cdot \mathbf{n}
$$

where $\mathbf{u}$ denotes the velocity vector. The PDFs of curvature and strain are shown in Fig. 5.
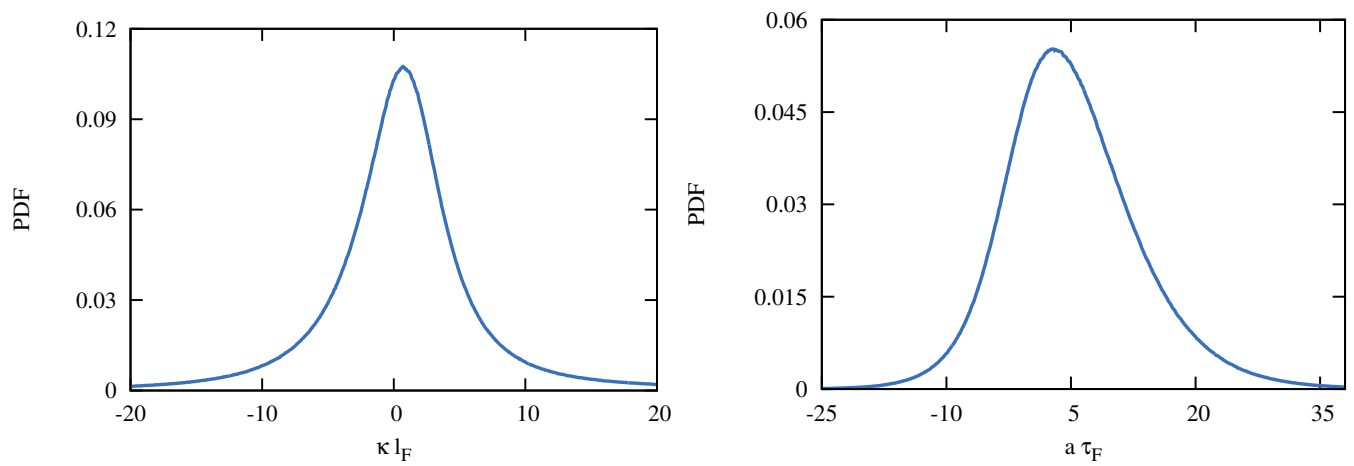

Figure 5: Left: PDF of the curvature. Right: PDF of the strain rate.

\subsubsection{Curvature effect}

Similar to the tangential diffusion term, the curvature is characterized by a zero mean and is almost symmetric around zero, as observed in Fig. 5. The curvature-conditioned average of the displacement speed is shown in Fig. 6 and it is observed that $s_{d}$ changes just slightly in the negative curvature range and increases with increasing positive curvature. High values of $s_{d}$ are associated with high curvature. This nonlinear behavior is mainly a response of the reaction term to curvature, as the profiles of $s_{d}$ and $V_{r}$ show a similar trend. The normal diffusion term is found to be nearly independent of curvature and, therefore, is not shown here. As seen in Eq. 5, the tangential diffusion term is a function only of the curvature. 

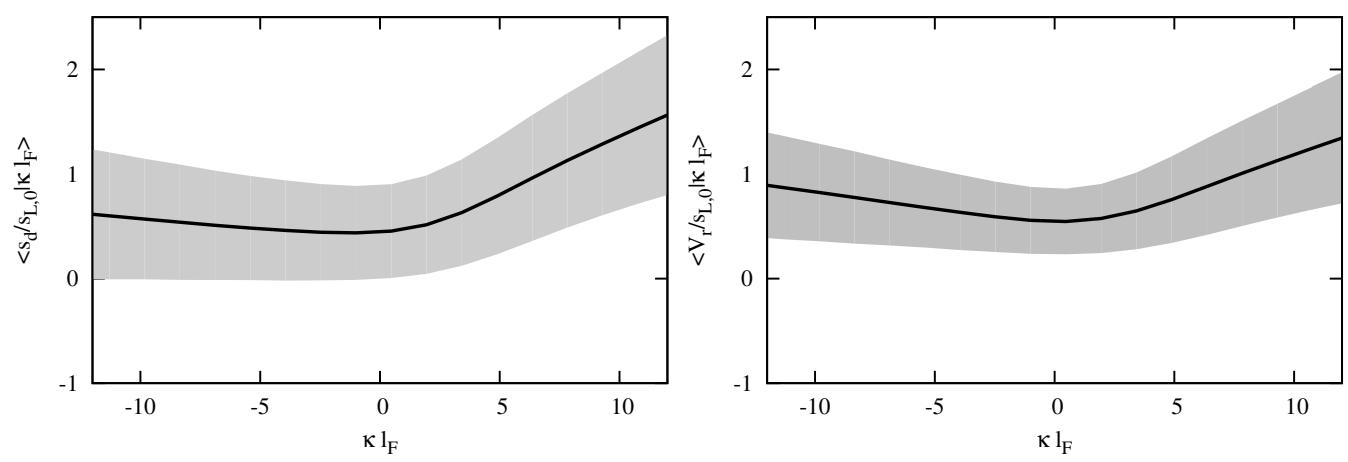

Figure 6: Response of displacement speed (left) and reaction term (right) to curvature. The gray area represents the conditional standard deviation.
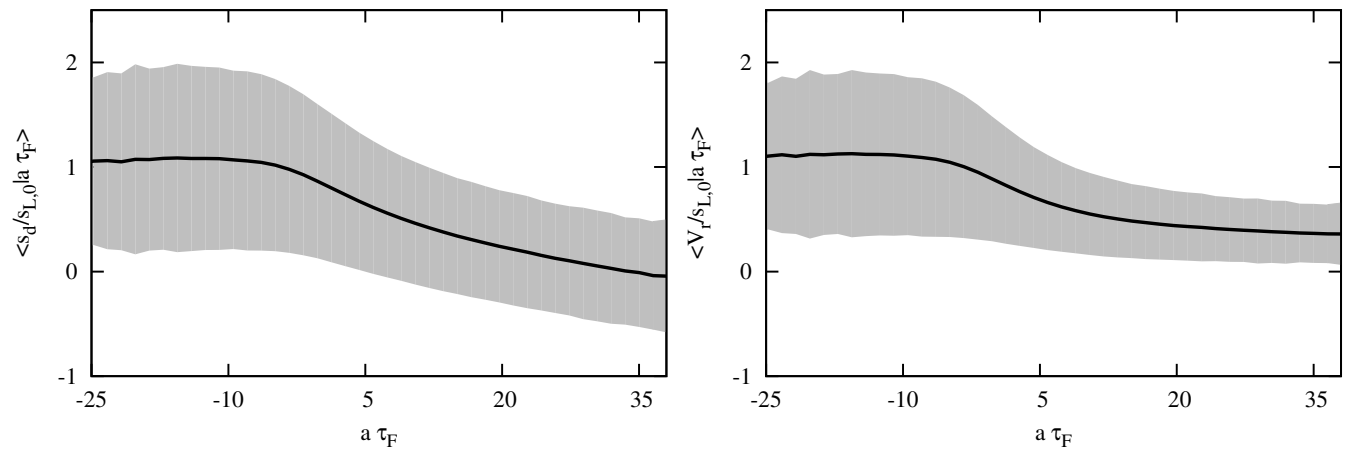

Figure 7: Response of displacement speed (left) and reaction term (right) to strain. The gray area represents the conditional standard deviation. 


\subsubsection{Strain effect}

First, it is noted that positive strain is found more frequently than negative strain (cf. Fig. 5). Its impact on $s_{d}$ is shown in Fig. 7 as the strain conditioned average of the displacement speed and three noteworthy observations are made. First, $s_{d}$ is nearly constant and equal to the laminar burning velocity for negative strain rates. Second, increasing positive strain rates lead to a noticeable decrease of the flame front displacement speed. The third point is that, analogously to the curvature effect, the change of $s_{d}$ due to strain is to leading order caused by the response of $V_{r}$ to strain. Based on these findings and along with the shape of the curvature and strain rate PDFs, it is concluded that strain is the driving mechanism for the flame to propagate slower than the laminar burning velocity.
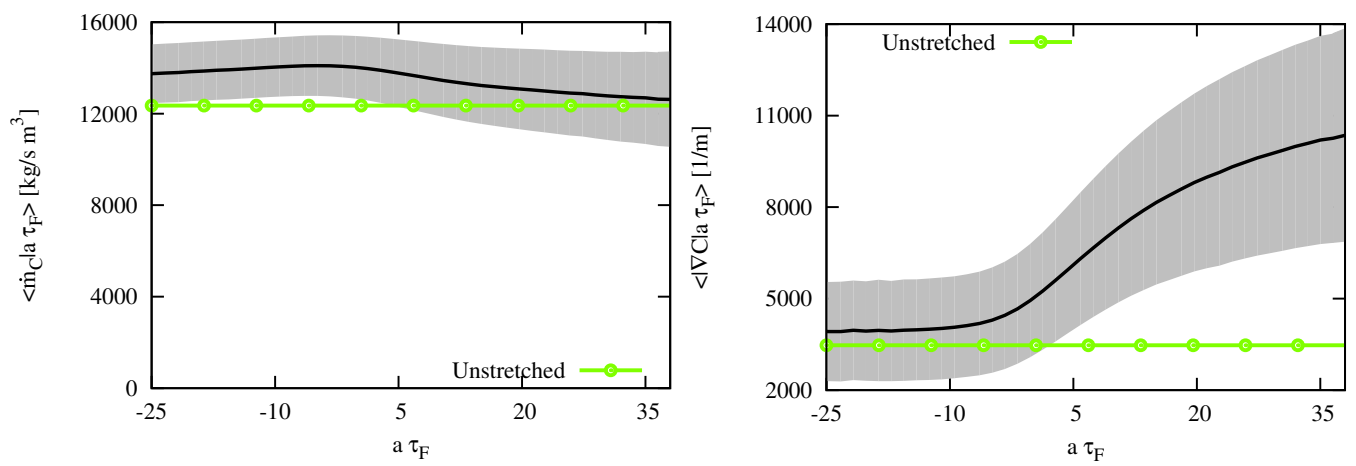

Figure 8: Response of the progress variable source term (left) and gradient (right) to strain. The gray area represents the conditional standard deviation.

In order to understand the effect of strain on $V_{r}$, the progress variable source term and gradient are plotted as a function of strain in Fig. 8 and contrasted to their equivalents from the unstretched flamelet solution. While the progress variable source term experiences at most $15 \%$ variations as a result of strain, the gradient gradually increases with increasing strain and reaches three times the value of an unstretched flame, which according to Eq. 8 then also leads to a strong decrease in $V_{r}$.

\subsubsection{Interdependencies between curvature and strain}

The two previous sections have shown that high $s_{d}$ values are found in positively curved flame pockets, which means they are curved into the burnt, while low displacement speeds originate from high strain rates. Both effects have been found to mainly affect the reaction term $V_{r}$. The fact that the mean 
flame front displacement speed is lower than the laminar burning velocity implies that the strain influence exceeds the curvature influence.

To further understand the interaction between these two competing effects, the joint PDF of curvature and strain is shown in Fig. 9. It may be

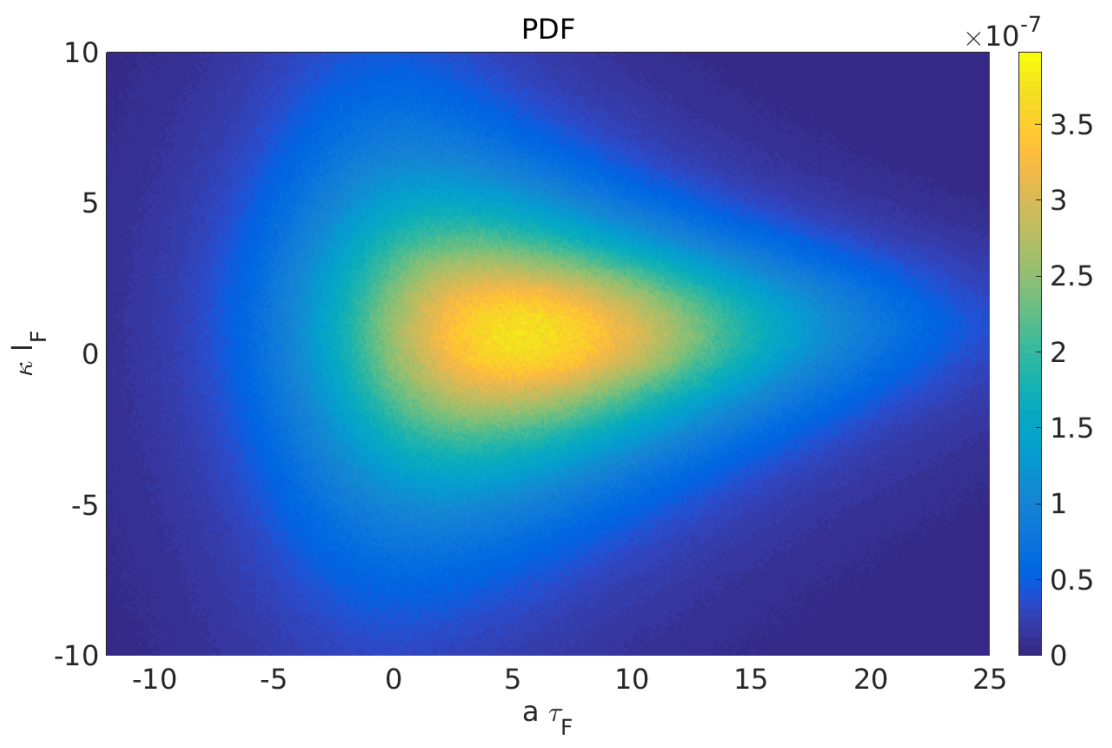

Figure 9: Joint PDF of curvature and strain. There is little coexistence of high curvature and high strain rates.

observed that the joint PDF is symmetric around zero curvature over the entire strain rate range. While the majority of flame elements are affected by both low to moderate curvature and strain, there is little coexistence of high strain and high curvature meaning that in the limits of very high or very low displacement speeds interdependencies between curvature and strain are unimportant.

\subsubsection{Generalized flame speed analysis}

Different definitions for the flame speed have been proposed in the literature. Poinsot and Veynante [35] have summarized different definitions and also discussed their implications. Throughout the present work, we have investigated the flame front displacement speed at a specific isosurface, which can be interpreted as a local quantity. However, in order to demonstrate the generality of the obtained results, we also analyze a more global quantity referred to as generalized flame speed [36]. The generalized flame speed it 
not limited to a given iso-surfaces and instead results from an integration over all iso-surfaces. Veynante and Vervisch [36] consider the relation

$$
\overline{\rho s_{d}|\nabla c|}=\rho_{u} \overline{\left(\frac{\rho}{\rho_{u}} s_{d}|\nabla c|\right)}=\rho_{u}\left\langle s_{d, u}\right\rangle_{s} \overline{|\nabla c|}
$$

to obtain the generalized flame speed with respect to the unburnt gases as

$$
\left\langle s_{d, u}\right\rangle_{s}=\frac{1}{\rho_{u}} \frac{\overline{\left(\rho s_{d}\right)|\nabla c|}}{\overline{|\nabla c|}}
$$

which is weighted with the flame surface density $|\nabla c|$. Note that the quantity $s_{d}$ appearing in the numerator of Eq. 13 is computed as outlined in Sect. 3.1. Here, the most important results are presented, especially the response of the generalized flame speed to the strain rate. Accordingly, the overbar operator appearing in Eqs. 12 and 13 denotes a mean conditioned on the strain rate. Although $\left\langle s_{d, u}\right\rangle_{s}$ can be evaluated as a field variable within the flame, it is obvious that some form of clipping is necessary, since $|\nabla c|$ becomes zero outside the flame zone. Therefore, the region between $C=0.1$ and $C=0.9$ is used for the analysis in this study.

In Fig. 10, the flame front displacement speed of $\mathrm{H}_{2} \mathrm{O}$ at the iso-surface $C=0.5$ and the generalized flame speed are compared. Specifically, both quantities and their contributions due to reaction, normal diffusion, and curvature are shown as a function of the strain rate. While the curvature terms remain almost constant around zero, the normal diffusion and reaction terms decrease with increasing strain rate for both flame speed quantities. Their decrease is responsible for the decrease of both the flame front displacement speed and the generalized flame speed. Moreover, both quantities are characterized by mean values of less than unity, which agrees with the conclusion from the flame front displacement speed analysis that the flame on average propagates slower than the laminar burning velocity. It is noted that the generalized flame speed features a very similar behavior as the flame front displacement speed, which largely confirms the results of Sect. 3.3. This is an interesting finding, since the flame front displacement speed and the generalized flame speed are quite different from a physical point of view. Nonetheless, the fact that both analyses result in the same conclusions confirms our findings and mutually supports both these perspectives. 


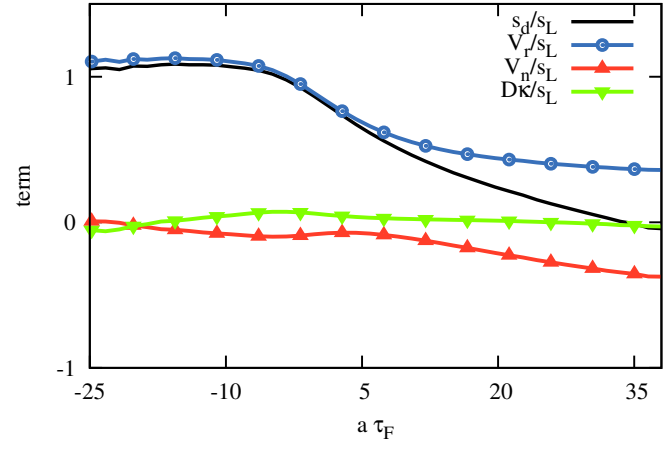

(a) Flame front displacement speed.

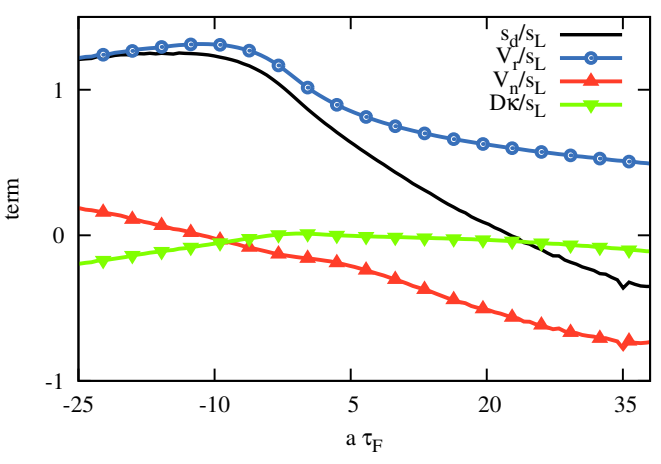

(b) Generalized flame speed.

Figure 10: Comparison of flame front displacement speed of $\mathrm{H}_{2} \mathrm{O}$ and generalized flame speed: Response of the generalized flame speed speed (black), reaction term (blue), the normal diffusion term (red), and the curvature term (green) to strain. All terms are normalized with the laminar burning velocity $s_{L}$.

\subsubsection{Implications for flame speed modeling}

Important conclusions of the displacement speed analysis in the context of modeling for the present case are the following:

- On average, the flame propagates slower than an unstretched laminar flame $\left\langle s_{d} / s_{L, 0}\right\rangle<1$.

- The influence of strain on the average flame speed is dominant over the influence of curvature.

- Strain causes $\left\langle s_{d} / s_{L, 0}\right\rangle<1$ via the reaction term $V_{r}$.

- The conditional mean $\left\langle s_{d} / s_{L, 0} \mid a \tau_{F}\right\rangle$ is constant and roughly unity for negative strain.

- The analyses of the flame front displacement speed and generalized flame speed result in the same conclusions and mutually support both these perspectives.

These conclusions imply that an accurate combustion model must predict a decreased local flame speed by accounting for strain effects. Moreover, it is shown that positive strain is much more likely to be found than negative strain. This finding, along with the insensitivity of the flame front displacement speed to negative strain rates, suggests that only positive strain has to 
be considered in modeling of the flame speed. Finally, it has been verified that the above outlined implications also hold for a flame front displacement speed analysis using hydrogen as the progress variable, which makes these conclusions for the present case general and independent of progress variable definition. The corresponding analysis is provided as part of the supporting material.

\section{Strained flamelet model}

Flamelet models map asymptotic combustion solutions into a flow field. These combustion solutions can be found using one-dimensional flamelets, which are solved prior to a simulation and accessed as needed during runtime. For this purpose, a simple way is to solve unstretched laminar flamelets and to use the progress variable as the mapping coordinate

$$
\phi_{k}=\mathcal{F}(C),
$$

where $\phi_{k}$ denotes any thermochemical quantity and $\mathcal{F}$ the functional relation governed by the unstretched premixed flamelet equations. As demonstrated in section 3, the influence of the strain rate component of stretch on the combustion process is not negligible. Note that other effects such as curvature or differential diffusion are not considered in the present model, since they are found to be unimportant on average, although they might be locally important.

The effect of strain is here modeled by the strained flamelet model of Knudsen et al. [13]. The underlying idea behind this model is to parameterize thermochemical quantities by the composition of a chemical species mass fraction, which represents the flame structure's internal response to strain. Furthermore, Knudsen et al. report, when applying such a strain parameterization, flamelet solutions from the back-to-back and fresh-to-burnt configurations collapse. This means that this technique eliminates the arbitrariness of selecting a particular strained flamelet configuration as long as the response itself is accurately mapped by the parameterizing species. In light of this finding, in this study back-to-back flamelets are considered and used to parameterize the modeling targets. The resulting characteristic relation between maximum temperature and strain rate, the so-called S-shaped curve, is shown in Fig. 11. These flamelet solutions are obtained using the FlameMaster program [37] utilizing a chemical mechanism for hydrogen [19] 


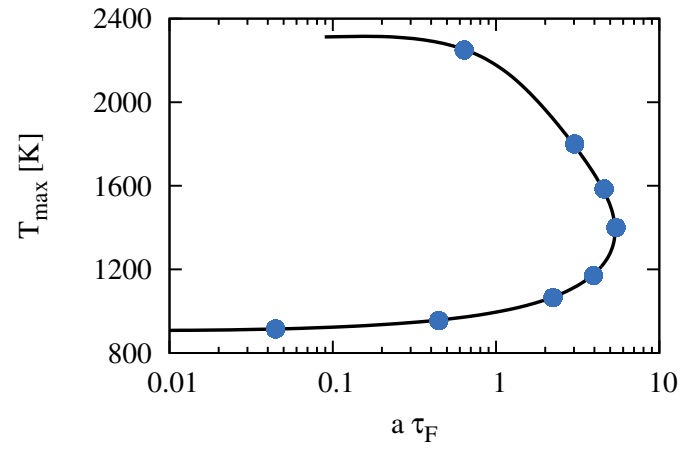

Figure 11: Premixed strained back-to-back flamelets. Maximum temperature over imposed strain rate. The dot symbols demarcate selected flamelet profiles that are shown in Fig. 12.

that consists of 9 species and 21 reactions and that has also been used in the DNS. As in the DNS, the Lewis numbers in the simulations for the strained flamelet model were non-unity and not constant.

The model of Knudsen et al. has been developed in an LES context. There are two important points to consider in LES models of the kind discussed here. The first is the model formulation and in particular the choice of correct parameters. The second is the LES formulation including the model for a presumed joint pdf. It is important to note here that the present paper is only concerned with the first point. The model formulation developed here could be used with any model for the joint pdf of the two parameters; it could even be used in combination with a transported PDF for the two parameters.

\subsection{Coordinate selection for strain parameterization}

In the strained flamelet model, a new parameter appears in the combustion model, which has to fulfill several criteria. The first one is that it is sensitive to strain. This means that a change of strain is accompanied by a change of the selected strain parameter. Additionally, the parameterization has to be unique in a sense that a movement along the curve shown in Fig. 11 leads to a monotonic variation of the species mass fraction representing strain. In order to minimize numerical errors associated with a table lookup, it is further desirable that the strain coordinate exhibits a significant variation and spans a wide range.

In Fig. 12, various flamelet profiles of three potential candidates including the mass fraction of the $\mathrm{H}$ radical chosen by Knudsen et al. [13] are shown 

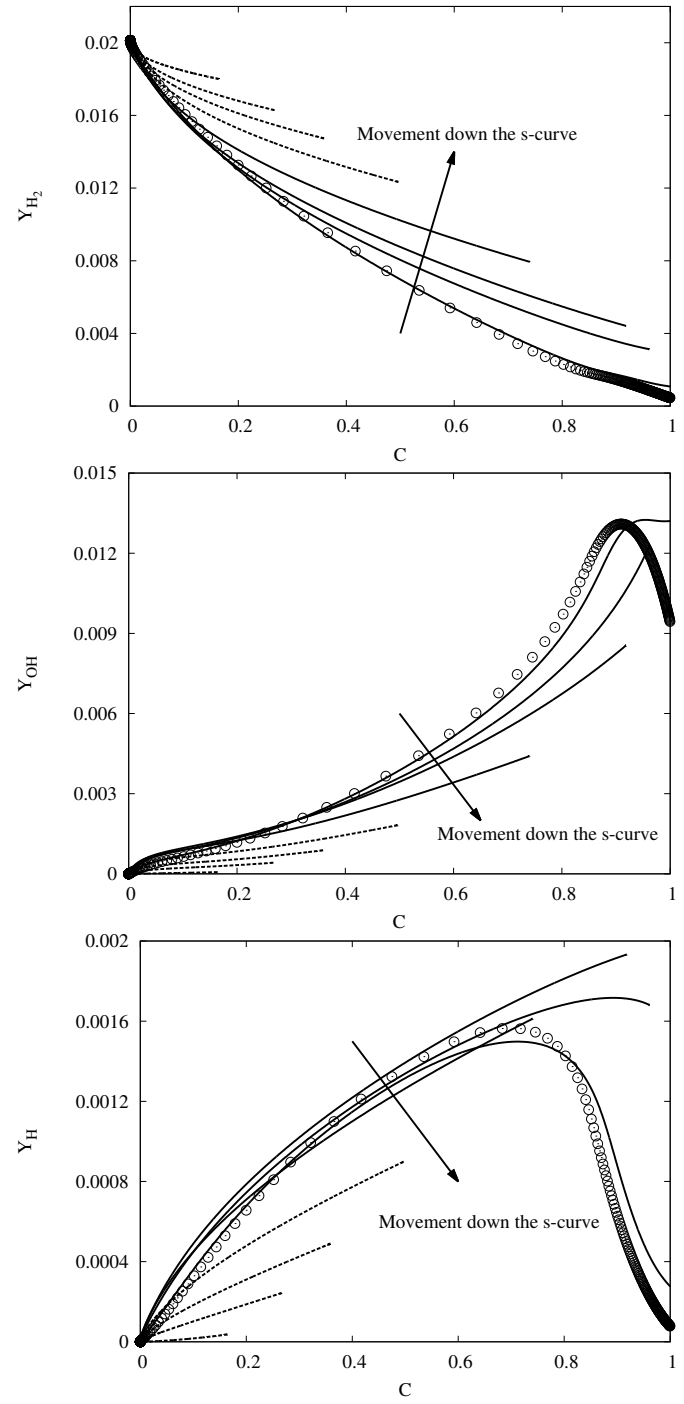

Figure 12: Response of selected species mass fraction to strain in premixed back-to-back flamelets. From top to bottom the mass fractions of $\mathrm{H}_{2}, \mathrm{OH}$, and $\mathrm{H}$ as function of $C$ and along the S-curve. The open circles are the unstretched flamelet solution, while solid and dotted lines are flamelet solutions of stable and unstable branch, respectively. The flamelet solutions correspond to the symbols in Fig. 11. 
as a function of the progress variable. It may be observed that the mass fractions $Y_{\mathrm{H}_{2}}, Y_{\mathrm{OH}}$, and $Y_{\mathrm{H}}$ alike are highly sensitive to strain and experience a notable change along the S-curve. These candidates span an almost equally wide range, although the respective absolute change of their compositions is different. In the present study, the mass fraction of $\mathrm{H}_{2}$ is chosen as the additional parameter, since $Y_{\mathrm{H}_{2}}$ is the only available species mass fraction with a strictly monotonic change. Both $Y_{\mathrm{H}}$ and $Y_{\mathrm{OH}}$ feature an overlap region between $C$ values of 0.8 and 0.9 implying that a parameterization on the basis of $Y_{\mathrm{OH}}$ and $Y_{\mathrm{H}}$ is not unique in this progress variable range. Thermochemical quantities are then given by

$$
\phi_{k}=\mathcal{G}\left(C, Y_{\mathrm{H}_{2}}\right),
$$

where $\mathcal{G}$ the functional relation governed by the premixed back-to-back flamelet equations. The selection of $Y_{\mathrm{H}_{2}}$ offers an additional advantage of implicitly accounting for differential diffusion effects, since its Lewis number is small. Finally, it is remarked that strained flamelets located on the top of the stable branch are almost identical with the unstretched flamelet. From this follows that the strained flamelets intrinsically include the unstretched solution.

\subsection{Modeling targets: Flame speed and reaction}

Two quantities, which are often required in combustion modeling, are the laminar burning velocity $s_{L}$ and the reaction source term $\dot{m}_{C}$. The laminar burning velocity is important as quantity to prescribe the propagation speed of the flame front, which is needed for example by the level set model, while the reaction source term is necessary for solving the transport equation of the progress variable. As pointed out by Knudsen et al. [13], one difference between unstretched and strained premixed flamelets is that the laminar burning velocity is not constant in strained solutions as it is in unstretched flamelets. Since the flame front displacement analysis is carried out at the isosurface $C=0.5$, the laminar burning velocity of the strained flamelets is extracted at this $C$ value.

In Fig. 13, both modeling targets are shown. The laminar burning velocity decreases from $8 \mathrm{~m} / \mathrm{s}$ for the unstretched flame to about $4.5 \mathrm{~m} / \mathrm{s}$ at the turning point between stable and unstable branch. Along the unstable branch of the S-curve, the laminar burning velocity reduces only slightly to around $4 \mathrm{~m} / \mathrm{s}$. The reaction source term is shown as a function of $C$ and $Y_{\mathrm{H}_{2}}$, where the lower bound of the $C-Y_{\mathrm{H}_{2}}$ space is confined by the unstretched flamelet 


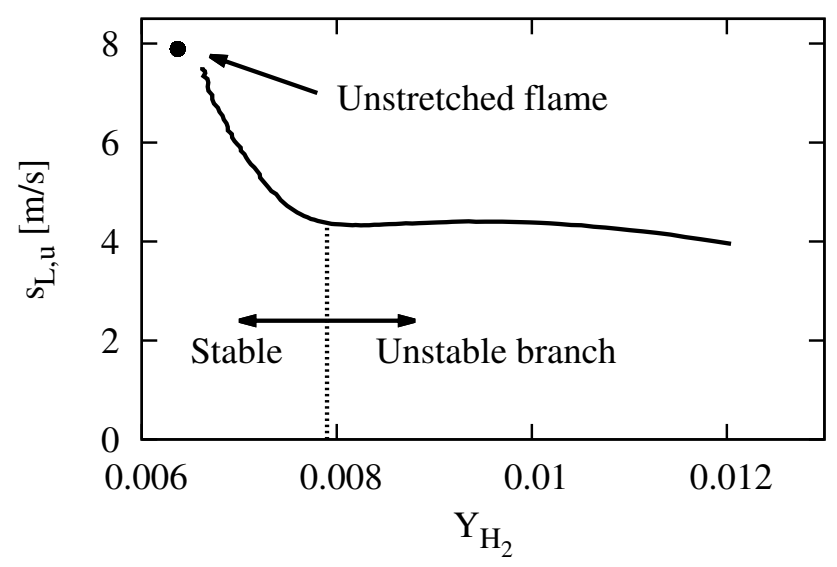

(a) Laminar burning velocity $s_{L, u}$ of strained flamelets

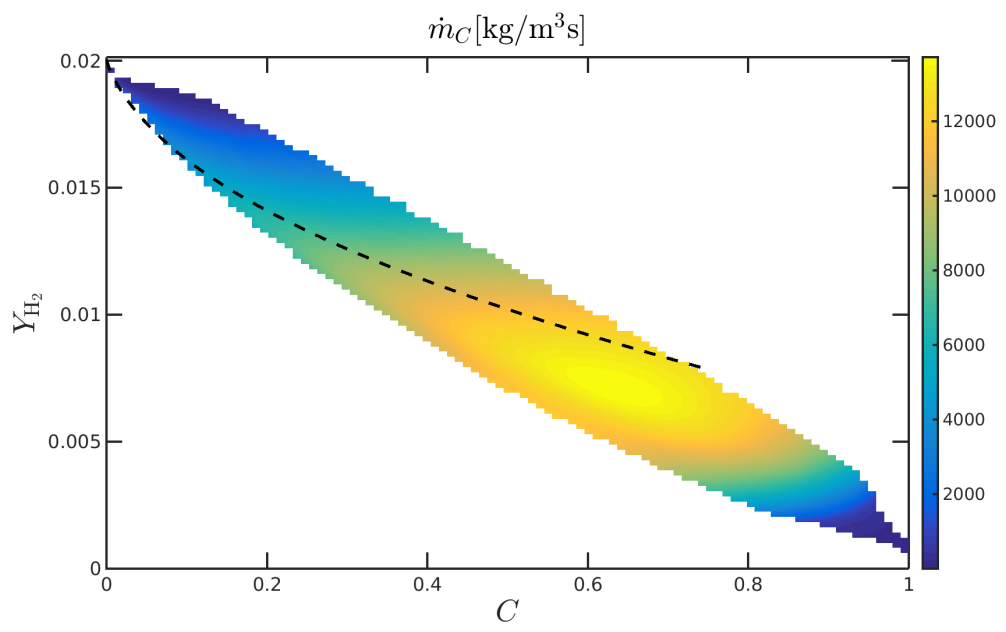

(b) Progress variable source term $\dot{m}_{C}$ from strained flamelet solutions

Figure 13: Top: Laminar burning velocity $s_{L, u}$ as a function of $Y_{\mathrm{H}_{2}}$. The black dot is the unstretched value. Bottom: Progress variable source term $\dot{m}_{C}$ as a function of $C$ and $Y_{\mathrm{H}_{2}}$. The dashed line demarcates the turning point of the S-shaped curve. 
solution. The progress variable source term $\dot{m}_{C}$ undergoes a significant variation with $Y_{\mathrm{H}_{2}}$ at a fixed $C$ corresponding to the water mass fraction, which means that strain strongly affects the source term. Note that the amount of variation is dependent on $C$; near the $C=0.5$ surface discussed earlier in the displacement speed analysis, the variations are about $15 \%$, but towards higher and lower $C$ values the effect of strain becomes more significant. It is interesting to observe that in the intermediate $C$ range, where the source term is largest, the maximum source term is found in strained flamelets of the stable branch and not in the unstretched solution.

\section{Model analysis}

In this section, the strained flamelet model is assessed using a priori testing. To this end, strained flamelet solutions of the flame speed and the progress variable source term are tabulated according to Eq. 15 and compared to the DNS and to the conventionally used unstretched premixed flamelet solutions given by Eq. 14 .

\subsection{Flame speed}

Fig. 14 shows the $\mathrm{PDF}$ of $Y_{\mathrm{H}_{2}}$ at the isosurface $\mathrm{C}=0.5$ and the conditional mean of $Y_{\mathrm{H}_{2}}$ over strain at $C=0.5$. According to the values of the hydrogen
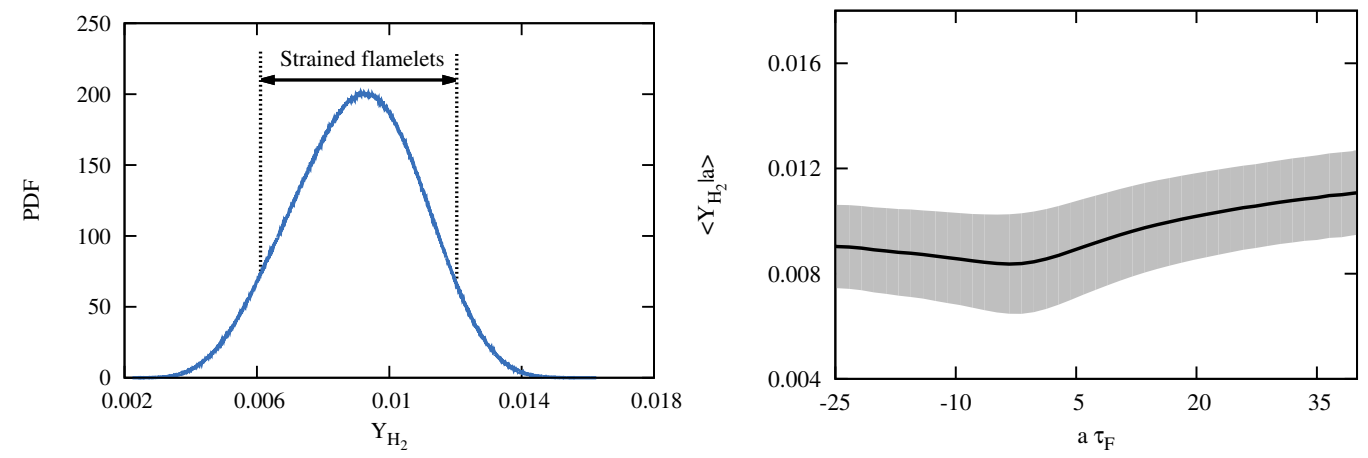

Figure 14: $\mathrm{PDF}$ of $Y_{\mathrm{H}_{2}}$ (left) and response of $Y_{\mathrm{H}_{2}}$ to strain at the flame front $C=0.5$. The strained flamelets are able describe around $90 \%$ of all points and positive strain leads to accumulation of $Y_{\mathrm{H}_{2}}$.

mass fraction observed in the DNS, strained flamelets cover around $90 \%$ of all points at this surface in the DNS and only the tails of the PDF fall outside the strained flamelet space. The response of the strain parameter $Y_{\mathrm{H}_{2}}$ 
selected here can be described as follows. Increasing positive strain results in an increase of $\mathrm{H}_{2}$ mass fraction, whereas decreasing negative strain rates lead to a slight increase of the $\mathrm{H}_{2}$ concentration. The behavior of $\mathrm{H}_{2}$ reveals a limitation of the strain modeling strategy followed here. In the DNS, high values of $Y_{\mathrm{H}_{2}}$ are found for both positive and negative strain rates, and since strained flamelet solutions only exist for positive strain rates, the strained flamelet model is not strictly able to distinguish between positive and negative strain rates. Here however, a good argument can be made that the $\mathrm{H}_{2}$ mass fraction is found to remain almost constant in the negative strain range and to vary significantly only for positive strain rates, similar to the situation with displacement speed.

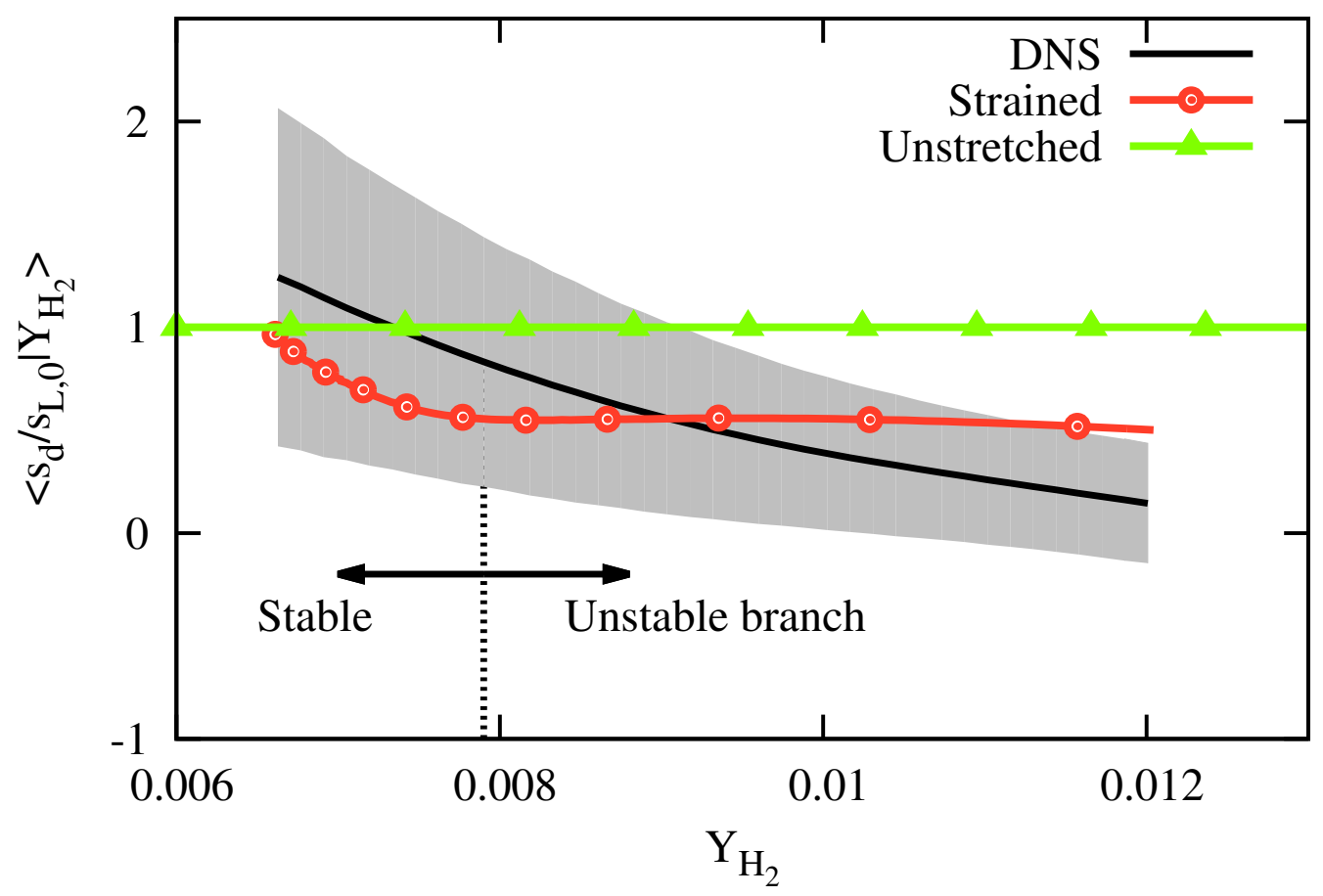

Figure 15: Modeling of the local flame speed. The black line is the conditional mean from the DNS where the gray area shows the conditional standard deviation. The red and green lines are the strained and unstretched flamelet model, respectively.

The model performance to predict the local flame speed is shown in Fig. 15. It may be observed that the strained flamelet model leads to improved predictions of the local flame speed, although the flame front displacement speed in the DNS decreases more rapidly than the modeled laminar 
burning velocity. In order to address the issue of partly misinterpreting positive and negative strain, the same analysis has been carried out for positive strain only, which is very similar to Fig. 15 and, therefore, is not shown here.

To understand the differences in the flame speeds between DNS and strained flamelets, the source term and the progress variable gradient as function of $Y_{\mathrm{H}_{2}}$ are shown in Fig. 16. The ratio of these quantities is the reaction
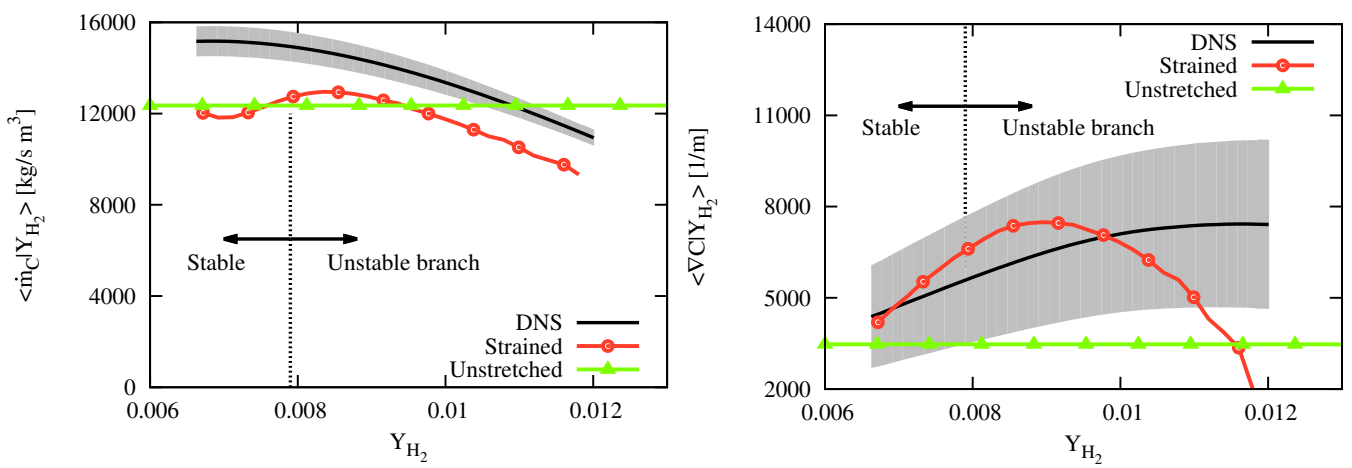

Figure 16: Progress variable source term (left) and gradient (right) as a function of $Y_{\mathrm{H}_{2}}$. The gray area shows the conditional standard deviation. The source term predictions are close to the DNS in terms of the sensitivity of the source term to $Y_{\mathrm{H}_{2}}$ variations, while the gradients differ significantly for higher $Y_{\mathrm{H}_{2}}$ concentration.

contribution $V_{r}$ of the flame speed, which has been identified in Sect. 3 as the main reason for the flame to interact with strain and to propagate slower than the laminar burning velocity. The source term in the DNS is higher than in both flamelet models, but the decline of $\dot{m}_{C}$ with increasing $Y_{\mathrm{H}_{2}}$ is captured quite accurately by the strained flamelet model. The increase of the progress variable gradient is predicted by the strained flamelet model up to an $\mathrm{H}_{2}$ mass fraction of $Y_{\mathrm{H}_{2}}=0.009$. However, beyond this value, the gradient in the strained flamelets falls off sharply, which is the reason why the flame speed predicted by the strained flamelet model remains almost constant at higher $Y_{\mathrm{H}_{2}}$ values and does not decrease further.

Finally, this result shows a further limitation of the strained flamelet model and more specifically of the unstable branch of the S-curve. Values up to $Y_{\mathrm{H}_{2}}=0.0079$ correspond to the stable branch, while values beyond this mass fraction are associated with the unstable branch. This means that the gradient behavior of flamelets of the unstable branch is opposed to the trend in the DNS as observed in the right panel of Fig. 16. One means of circumventing this problem is the model extension towards an unsteady 
strained flamelet model, which could be done in future work.

\subsection{Reaction source term}
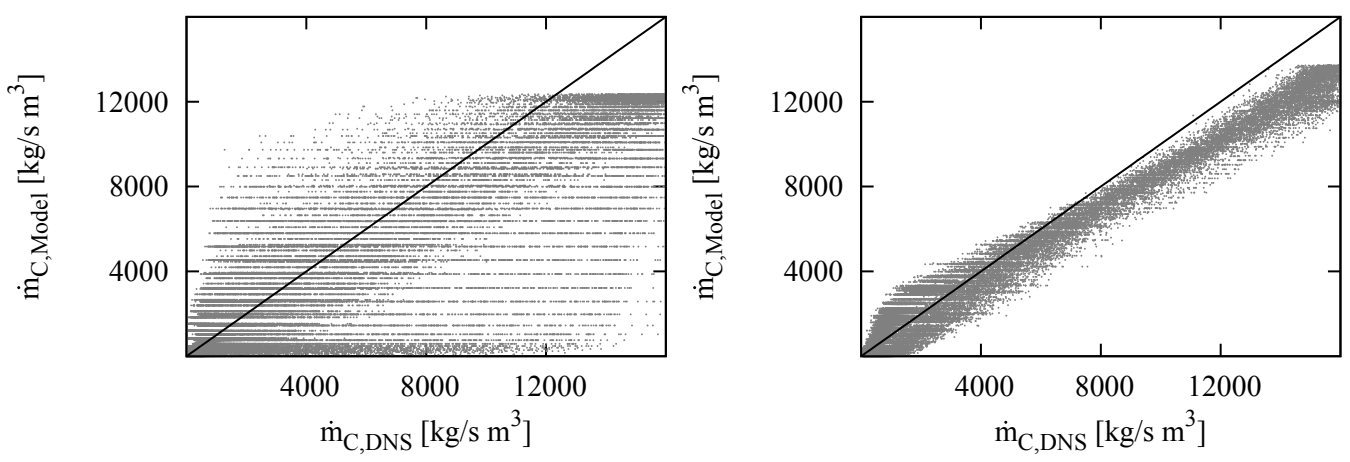

Figure 17: Scatter plots of reaction source term from DNS and from unstretched (left) and strained flamelet model (right). In the scatter plots, the line is a perfect model. The strained flamelet model removes much of the scatter of the unstretched flamelet model.

Figure 17 shows scatter plots of the reaction source term closure for the unstretched and strained flamelet models. For the unstretched model, an immense amount of scatter is observed, whereas most of this scatter disappears in the strained model. This means that the additional information provided by the strained flamelets improves the source term closure significantly. While the scatter plots allow for a visual evaluation of the modeling strategy, they do not provide further quantitative information. This is obtained here using the optimal estimator approach. Before results from this analysis methodology are presented, the optimal estimator approach is briefly reviewed.

For this, we define the total error of a model with respect to the DNS data as

$$
\epsilon_{\mathrm{tot}}\left(C, Y_{\mathrm{H}_{2}}\right)=\left\langle\left(\dot{m}_{C, \mathrm{DNS}}-\dot{m}_{C, \text { Model }}\left(C, Y_{\mathrm{H}_{2}}\right)\right)^{2}\right\rangle,
$$

where $\dot{m}_{C, \mathrm{DNS}}$ is the progress variable source term directly evaluated from the DNS, $\dot{m}_{C, \text { Model }}\left(C, Y_{\mathrm{H}_{2}}\right)$ is the model evaluated with the parameters $C$ and $Y_{\mathrm{H}_{2}}$ from the DNS, and the $\langle\cdot\rangle$ operator denotes an average conditioned on $C$ and $Y_{\mathrm{H}_{2}}$. The error due to the selection of the set of parameters, the so called irreducible error, is given as

$$
\epsilon_{\mathrm{irr}}\left(C, Y_{\mathrm{H}_{2}}\right)=\left\langle\left(\dot{m}_{C, \mathrm{DNS}}-\left\langle\dot{m}_{C} \mid\left(C, Y_{\mathrm{H}_{2}}\right)\right\rangle\right)^{2}\right\rangle,
$$


where the conditional mean $\left\langle\dot{m}_{C} \mid\left(C, Y_{\mathrm{H}_{2}}\right)\right\rangle$ appearing in this expression is referred to as the optimal estimator and is the best possible model result that one could achieve with this set of parameters. The difference between $\epsilon_{\mathrm{tot}}$ and $\epsilon_{\mathrm{irr}}$ is the remaining error that stems from the functional form of the model for $\dot{m}_{C}$.

While for the unstretched model, $C$ is the only parameter, the strained flamelet model parametrizes $\dot{m}_{C}$ with respect to $C$ and $Y_{\mathrm{H}_{2}}$ so that its errors are also given in terms of $C$ and $Y_{\mathrm{H}_{2}}$. In order to compare the errors of both models directly, those of the strained flamelet model are weighted with the conditional PDF of $Y_{\mathrm{H}_{2}}$ at a given value of the progress variable $P\left(Y_{\mathrm{H}_{2}} \mid C\right)$. Three different forms of the irreducible and total model errors are introduced and discussed here. On the one hand, the errors are normalized by the maximum conditional mean of the progress variable source term $\left\langle\dot{m}_{C} \mid C\right\rangle_{\max }$ to obtain a non-dimensional absolute model error. On the other hand, the errors are normalized by the optimal estimator $\left\langle\dot{m}_{C} \mid C\right\rangle$ at a fixed $C$, which can be interpreted as an error relative to the optimal estimator. The absolute and relative errors, for example of the total model error, are then given by

$$
\epsilon_{\mathrm{tot}, C, \mathrm{abs}}=\int P\left(Y_{\mathrm{H}_{2}} \mid C\right) \epsilon_{\mathrm{tot}}\left(C, Y_{\mathrm{H}_{2}}\right) \mathrm{d} Y_{\mathrm{H}_{2}} /\left\langle\dot{m}_{C} \mid C\right\rangle_{\max }^{2}
$$

and

$$
\epsilon_{\mathrm{tot}, C, \mathrm{rel}}=\int P\left(Y_{\mathrm{H}_{2}} \mid C\right) \epsilon_{\mathrm{tot}}\left(C, Y_{\mathrm{H}_{2}}\right) \mathrm{d} Y_{\mathrm{H}_{2}} /\left\langle\dot{m}_{C} \mid C\right\rangle^{2} .
$$

Finally, an absolute error density can be defined by weighting the absolute error with the PDF of $C$ as

$$
\epsilon_{\mathrm{tot}, C, \mathrm{den}}=P(C) \epsilon_{\mathrm{tot}, C, \text { abs }}
$$

to quantify, how different intervals of the progress variable contribute to the accumulated net error of the source term closure. The integral of this error density over a range of progress variable values then corresponds to the total absolute error caused by the model error in that range of progress variable values.

In Fig. 18, the irreducible and the total model errors of both models are shown in their relative, absolute, and weighted form. From a comparison of the irreducible errors follows that the $\mathrm{H}_{2}$ mass fraction contains additional physical information for the source term closure that allows to decrease the error by one order of magnitude. The relative irreducible errors show a minimum around $C=0.5$ in the inner layer region, while the absolute irreducible 

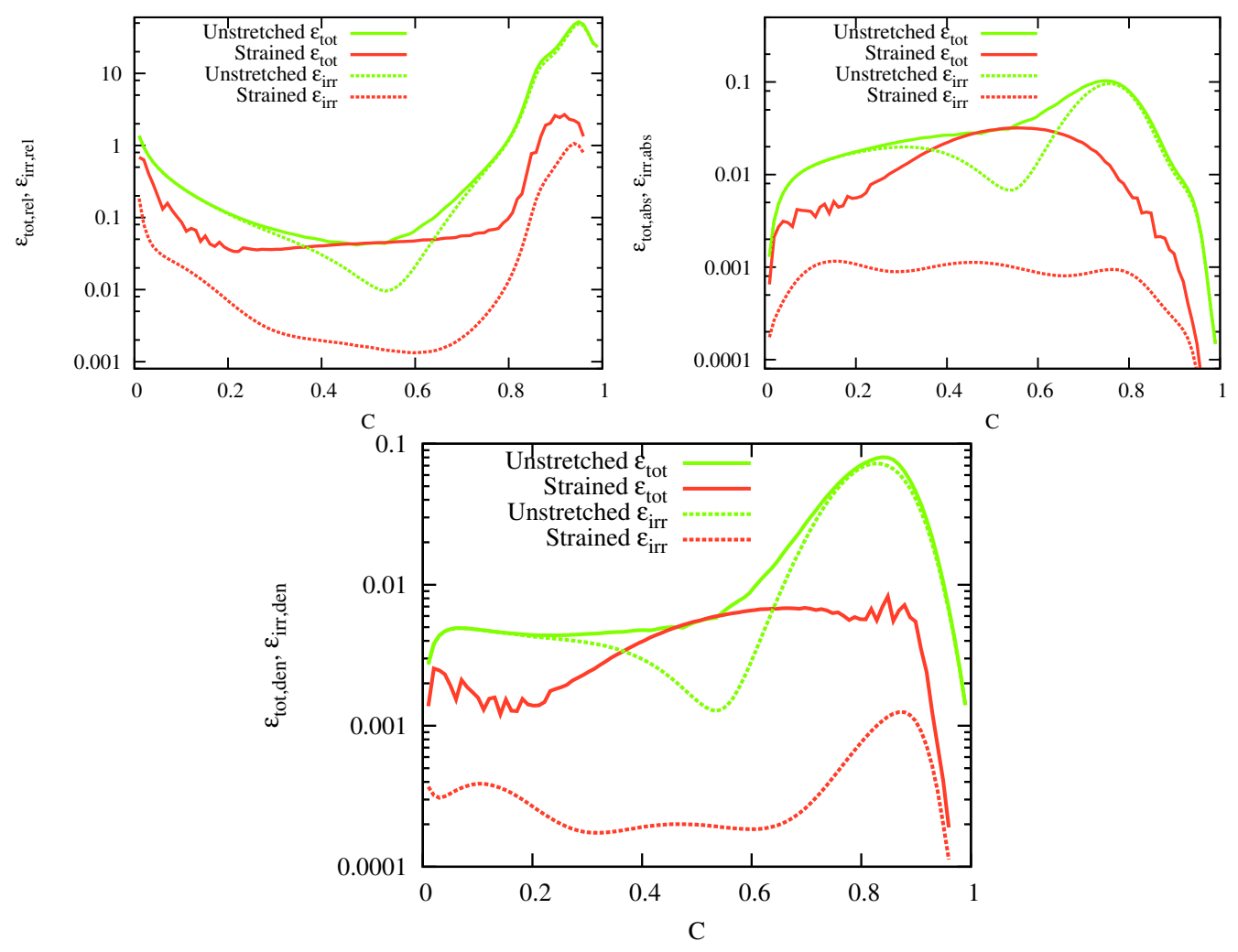

Figure 18: In the top row, the irreducible and total model errors of the unstretched and strained flamelet model are shown. The relative errors are shown on the left, while the absolute errors are shown on the right. Error densities are shown in the bottom row. 
errors peak around the inner layer region. The profiles of the total model error of the reaction source term indicate that the model error of the strained model is much lower than the model error of the unstretched model for all progress variable values except a narrow intermediate progress variable region. Moreover, the model error of the strained flamelet model is close to the irreducible error for very high and very low $C$ values. The source term closure is particularly improved around $C=0.8$, where the absolute error of the unstretched model peaks. On the contrary, the absolute total model error of the strained flamelet model is one order of magnitude lower in this progress variable range. This is beneficial for the prediction of the total heat release, since this range contributes most to the total model error density of the unstretched model, whereas the error reduction around $C=0.8$ leads to largely reduced integrated total model error of the strained model. In summary, these findings directly translate to the scatter plots (see Fig. 17) of the source term closure and demonstrate that the strained flamelet model is a significant improvement over the unstretched flamelet model.

Finally, the effect of curvature and strain on the source term closure is investigated. While strain effects are modeled by the strained flamelet model, the influence of curvature is not considered. In order to assess the consequence of this simplification on the source term closure, source term error, i.e. the difference between the source term from the DNS and the respective

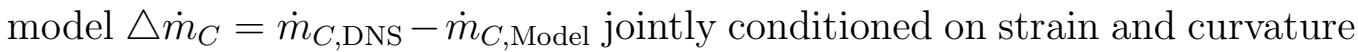
is shown in Fig. 19. At low curvature and high strain rates, the unstretched flamelet model underpredicts the reaction source term, which yields a peak error for such a stretch scenario. This error completely disappears in case of the strained flamelet model. From this follows that model errors due to strain are to leading order eliminated by the strained flamelet model. Interestingly, the strained flamelet model also reduces the model error in the negative strain rate range, although the strained flamelet solutions feature only positive strain.

\subsection{Model evaluation during flame evolution}

The analyses presented so far were carried out at the normalized time $t / t_{j}=17.1$, as this is the instant of strongest turbulence-chemistry interactions. Since the investigated flame is temporally developing, the difference between the heat release and flame surface area and the behavior of $I_{0}$ in Fig. 2 might also arise from effects of unsteadiness apart from turbulencechemistry interactions. It is therefore essential to evaluate the model at all 


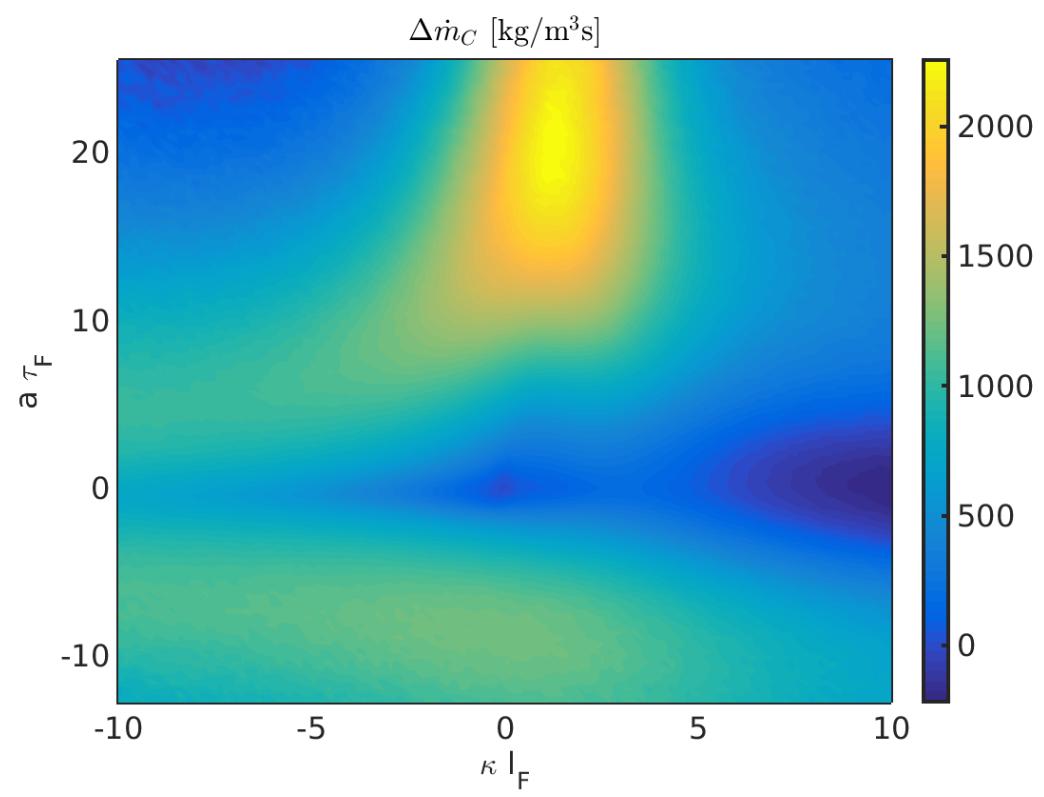

(a) Unstretched flamelet model.

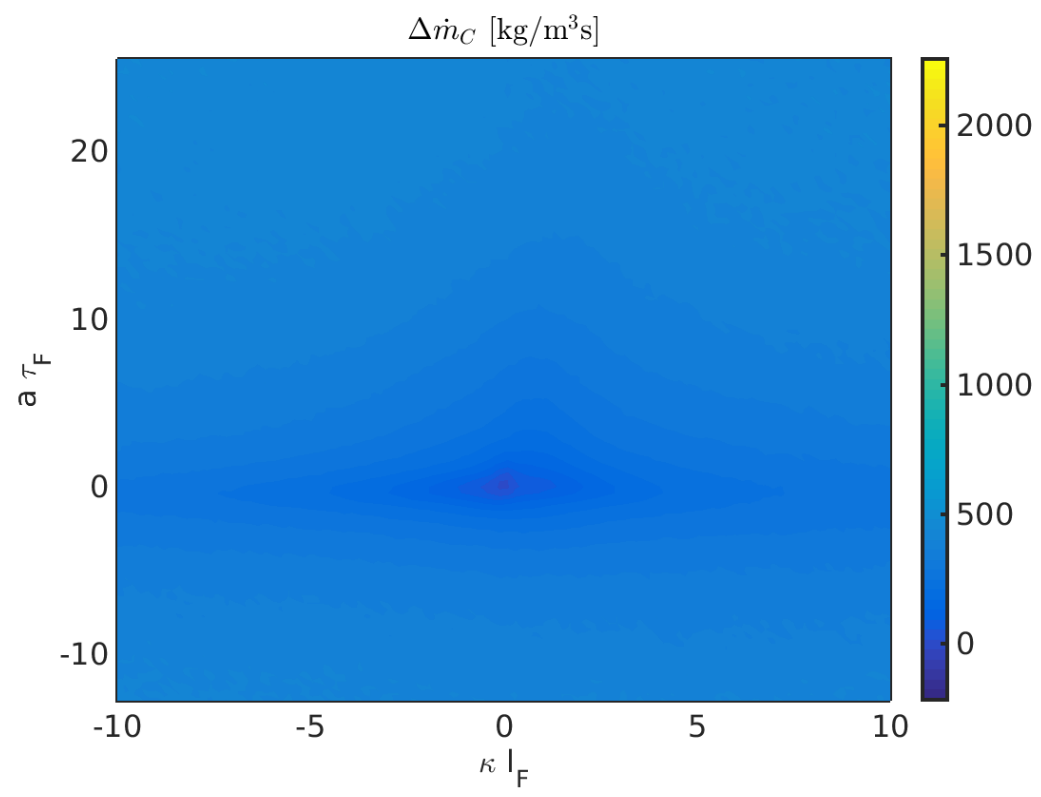

(b) Strained flamelet model.

Figure 19: Modeling error of reaction source term conditioned on curvature and strain. Top: Unstretched flamelet model. Bottom: Strained flamelet model. In both figures, on the horizontal axis is curvature and on the vertical axis strain. 
relevant simulation times, especially because the flame is modeled with a strained flamelet model that relies on steady flamelet solutions.

To this end, both the unstretched and strained flamelet model are employed to compute the production speed $s_{p, \mathrm{H}_{2} \mathrm{O}}$ that is then compared to the one measured in the DNS. The progress variable source term of both models is given by Eq. 14 and Eq. 15, respectively, and integrated according to Eq. 2 to obtain $s_{p, \mathrm{H}_{2} \mathrm{O}}$. While this calculation can be straightforwardly executed for the unstretched flamelet model, a treatment is needed for flame elements which fall out of the flamelet space in case of the strained flamelet model. Here, a simple strategy is suggested by using the solution that is closest to such elements in $C$ space. The comparison of $s_{p, \mathrm{H}_{2} \mathrm{O}}$ shown in Fig. 20 is omitted for early simulation times, when the flame is still fully laminar and undisturbed by the turbulence. Concurring with Sect. 5.2, the strained flamelet model performs significantly better than the unstretched model and only slightly overpredicts the production speed for late simulation times.

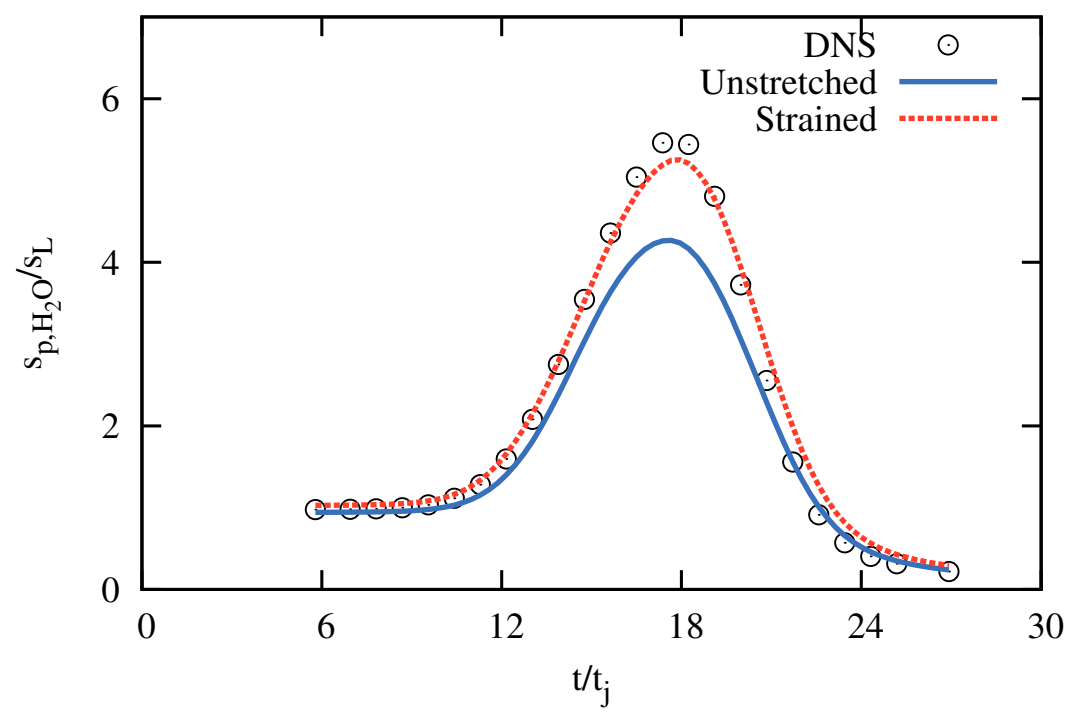

Figure 20: Comparison of the integrated production rate of $\mathrm{H}_{2} \mathrm{O}$ measured in the DNS and predicted by both models. Note that early simulation times are not included.

\section{Conclusion}

In this work, turbulence-chemistry interactions were analyzed and modeled for lean premixed turbulent hydrogen flames. Capabilities and limita- 
tions of an unstretched and a strained premixed flamelet model to describe turbulent premixed flames in the thin reaction zones regime were discussed with respect to the modeling targets, the flame speed and the reaction rate of the progress variable. To this end, finite-rate chemistry effects were investigated by means of a flame front displacement analysis. Based on this analysis, the interaction of turbulence and chemistry due to flame stretch is modeled with a strained flamelet model, which was validated against the DNS data.

The flame front displacement analysis splits the total flame displacement speed into three contributions stemming from reaction, normal diffusion, and tangential diffusion. Statistics of the flame front displacement speed and its contributions were presented and it was found that the overall displacement speed is lower than in the laminar unstretched case. In order to identify the origin of this behavior, the effect of flame stretch on the displacement speed was analyzed. It was demonstrated that the response of the flame front displacement speed is mainly attributed to a change in the reaction contribution. High displacement speed corresponds to high curvature, while low displacement speed is associated with high strain. Overall, strain effects dominate curvature effects so that the global flame speed is lower than the laminar burning velocity, and make turbulence-chemistry interactions in form of strain the modeling topic of the present study.

Following the ideas of Knudsen et al. [13], strain effects are considered here using a strained flamelet model. This model incorporates strained flamelet solutions from the back-to-back strained premixed laminar flame configuration and captures the flame structure's internal response to strain by the response of a chemical component to strain. Here, the fuel mass fraction, $Y_{\mathrm{H}_{2}}$, was found to vary strictly monotonically along the S-curve, and thus to be appropriate to parameterize strain. $Y_{\mathrm{H}_{2}}$ as the strain marker along with the conventionally used progress variable were then used to parameterize the modeling targets.

Both the unstretched and strained premixed flamelet model were a priori analyzed against the DNS data. First, their ability to predict the local flame speed was compared and it was demonstrated that the strained model leads to a reasonable improvement of flame speed predictions. Remaining discrepancies between DNS and the strained flamelet model originate from differences of the progress variable gradient, which is much lower in the model than in the DNS. The second modeling part is devoted to the reaction rate of the progress variable. The optimal estimator approach was applied and in- 
dicated that the irreducible error is significantly decreased by the strained in comparison to the unstretched flamelet model. It was further shown that the determination of the progress variable source term greatly benefits from the strained flamelet model and, in particular, eliminates model errors occurring at high strain rates. Finally, the model performance during the evolution of the flame was evaluated by comparing the measured and predicted values of the production speed of $\mathrm{H}_{2} \mathrm{O}$. This comparison revealed that the strained flamelet model significantly improves the closure of this quantity and is able to predict the DNS curve very accurately.

In summary, findings from the present study support the modeling strategy of Knudsen et al. [13] and encourage the application of the proposed strained flamelet model in simulations of highly turbulent premixed flames, where it is also shown that the strain parameterization is different for hydrogen flames. However, it is important to recognize that the specific fuel and fresh gas conditions adopted here leads to a reaction zone that is relatively broad compared with that in a hydrocarbon flame. This might have important effects, and further investigations should be performed for hydrocarbon flames in the future.

\section{Acknowledgment}

This research was performed as part of the collaborative research center SFB 686, which is funded by the German Research Foundation (Deutsche Forschungsgemeinschaft (DFG)). E. R. Hawkes is supported by the Australian Research Council. This support is gratefully acknowledged. Computer resources for this project have been provided by the Gauss Centre for Supercomputing/Leibniz Supercomputing Centre under grant pr45di and by the National Center for Computational Science at Oak Ridge National Laboratory (NCCS/ORNL), which is supported by the Office of Science of the DOE under Contract No. DE-AC05-00OR22725. The authors would like to thank Dr. Haiou Wang for his help in transferring the DNS data from Oak Ridge to Aachen.

[1] S. B. Pope, PDF methods for turbulent reactive flows, Prog. Energy Combust. Sci. 11 (1985) 119-192.

[2] O. Colin, D. Veynante, T. Poinsot, A thickened flame model for large eddy simulation of premixed turbulent combustion, Phys. Fluids 12 (2000) 1843-1863. 
[3] A. Kerstein, Linear eddy modelling of turbulent transport. Part 7. Finite-rate chemistry and multi-stream mixing, J. Fluid Mech. 240 (1992) 289-313.

[4] R. W. Bilger, Conditional moment closure for turbulent reacting flow, Phys. Fluids A 5 (1993) 436-444.

[5] N. Peters, Turbulent Combustion, Cambridge University Press, Cambridge, UK, 2000.

[6] M. Boger, D. Veynante, H. Boughanem, A. Trouvé, Direct numerical simulation analysis of flame surface density concept for large eddy simulation of turbulent premixed combustion, Proc. Combust. Inst. 27 (1998) 917-925.

[7] E. R. Hawkes, R. S. Cant, A flame surface density approach to largeeddy simulation of premixed turbulent combustion, Proc. Combust. Inst. 28 (2000) 51-58.

[8] J. A. Oijen, F. A. Lammers, L. P. H. de Goey, Modeling of complex premixed burner systems by using flamelet-generated manifolds, Combust. Flame 127 (2001) 2124-2134.

[9] O. Gicquel, N. Darabiha, D. Thévenin, Laminar premixed hydrogen/air counterflow flame simulations using flame propagation of ILDM with preferential diffusion, Proc. Combust. Inst. 28 (2000) 1901-1908.

[10] N. Peters, The turbulent burning velocity for large-scale and small-scale turbulence, J. Fluid Mech. 384 (1999) 107-132.

[11] J. A. Oijen, L. P. H. de Goey, Modelling of premixed counterflow flames using the flamelet-generated manifold method, Combust. Theor. Model. 6 (2002) 463-478.

[12] J. A. Oijen, R. J. M. Bastiaans, L. P. H. de Goey, Low-dimensional manifolds in direct numerical simulations of premixed turbulent flames, Proc. Combust. Inst. 31 (2007) 1377-1384.

[13] E. Knudsen, H. Kolla, E. R. Hawkes, H. Pitsch, LES of a premixed jet flame DNS using a strained flamelet model, Combust. Flame 160 (2013) 2911-2927. 
[14] E. R. Hawkes, J. H. Chen, Comparison of direct numerical simulation of lean premixed methane-air flames with strained laminar flame calculations, Combust. Flame 144 (2006) 112-125.

[15] H. Kolla, N. Swaminathan, Strained flamelets for turbulent premixed flames II: Laboratory flame results, Combust. Flame 157 (2010) 12741289 .

[16] E. R. Hawkes, O. Chatakonda, H. Kolla, A. R. Kerstein, J. H. Chen, A petascale direct numerical simulation study of the modelling of flame wrinkling for large-eddy simulations in intense turbulence, Combust. Flame 159 (2012) 2690-2703.

[17] O. Chatakonda, E. R. Hawkes, A. J. Aspden, A. R. Kerstein, H. Kolla, J. H. Chen, On the fractal characteristics of low Damköhler number flames, Combust. Flame 159 (2013) 2690-2703.

[18] J. H. Chen, A. Choudhary, B. D. Supinski, M. DeVries, E. R. Hawkes, S. Klasky, W. K. Liao, K. L. Ma, J. Mellor-Crummey, N. Podhorszki, R. Sankaran, S. Shende, C. S. Yoo, Terrascale direct numerical simulations of turbulent combustion using S3D, Comput. Sci. Discovery 2 (2009) Art. No. 015001.

[19] J. Li, Z. Zhao, A. Kazakov, F. L. Dryer, An updated comprehensive kinetic model of hydrogen combustion, Int. J. Chem. Kinet. 36 (2004) $566-575$.

[20] E. Knudsen, S. H. Kim, H. Pitsch, An analysis of premixed flamelet models for large eddy simulation of turbulent combustion, Phys. Fluids 22 (2010) Art. No. 115109.

[21] K. N. C. Bray, R. S. Cant, Some Applications of Kolmogorov's Turbulence Research in the Field of Combustion, Proc. Roy. Soc. Lond. A 434 (1991) 217-240.

[22] C. H. Gibson, Fine Structure of Scalar Fields Mixed by Turbulence. I. Zero-Gradient Points and Minimal Gradient Surfaces, Phys. Fluids (1958-1988) 11 (1968) 2305-2315.

[23] S. B. Pope, The evolution of surfaces in turbulence, Int. J. Eng. Sci. 26 (1988) 445-469. 
[24] T. Echekki, J. H. Chen, Analysis of the contribution of curvature to premixed flame propagation, Combust. Flame 118 (1999) 308-311.

[25] N. Peters, P. Terhoeven, J. H. Chen, T. Echekki, Statistics of flame displacement speeds from computations of 2-D unsteady methane-air flames, Symposium (International) on Combustion 27 (1998) 833-839.

[26] J. H. Chen, H. G. Im, Stretch effects on the burning velocity of turbulent premixed hydrogen/air flames, Proc. Combust. Inst. 28 (2000) 211-218.

[27] E. R. Hawkes, J. H. Chen, Direct numerical simulation of hydrogenenriched lean premixed methane-air flames, Combust. Flame 138 (2004) 242-258.

[28] N. Chakraborty, R. S. Cant, Unsteady effects of strain rate and curvature on turbulent premixed flames in an inflow-outflow configuration, Combusti. Flame 137 (2004) 129-147.

[29] E. R. Hawkes, J. H. Chen, Evaluation of models for flame stretch due to curvature in the thin reaction zones regime, Proc. Combust. Inst. 30 (2005) 647-655.

[30] N. Chakraborty, M. Klein, R. S. Cant, Stretch rate effects on displacement speed in turbulent premixed flame kernels in the thin reaction zones regime, Proc. Combust. Inst. 31 (2007) 1385-1392.

[31] N. Chakraborty, E. R. Hawkes, J. H. Chen, R. S. Cant, The effects of strain rate and curvature on surface density function transport in turbulent premixed methan-air and hydrogen-air flames: A comparative study, Combust. Flame 154 (2008) 259-280.

[32] I. Han, K. Y. Huh, Effects of the Karlovitz number on the evolution of the flame surface density in turbulent premixed flames, Proc. Combust. Inst. 32 (2009) 1419-1425.

[33] C. Altantzis, C. E. Frouzakis, A. G. Tomboulides, M. Matalon, K. Boulouchos, Hydrodynamic and thermodiffusive instability effects on the evolution of laminar planar lean premixed hydrogen flames, J. Fluid Mech. 700 (2012) 329-361. 
[34] R. Sankaran, E. R. Hawkes, C. S. Yoo, J. H. Chen, Response of flame thickness and propagation speed under intense turbulence in spatially developing lean premixed methane-air jet flames, Combust. Flame 162 (2015) 3294-3306.

[35] T. Poinsot, D. Veynante, Theoretical and Numerical Combustion, R. T. Edwards Inc., Philadelphia, USA, 2005.

[36] D. Veynante, L. Vervisch, Turbulent combustion modeling, Prog. Energy Combust. Sci. 28 (2002) 192-266.

[37] H. Pitsch, A C ++ computer program for 0D and 1D laminar flame calculations, http://http://www.itv.rwthaachen.de/downloads/flamemaster/, 1998. 